\title{
Renormalization of gauge theories in the background-field approach
}

\author{
Andrei O. Barvinsky, ${ }^{a}$ Diego Blas, ${ }^{b}$ Mario Herrero-Valea, ${ }^{c}$ Sergey M. Sibiryakov ${ }^{b, c, d}$ \\ and Christian F. Steinwachs ${ }^{e}$
}

${ }^{a}$ Theory Department, Lebedev Physics Institute, Leninsky Prospect 53, Moscow 119991, Russia

${ }^{b}$ Theoretical Physics Department, CERN,

CH-1211 Geneva 23, Switzerland

${ }^{c} L P P C$, Institute of Physics, EPFL, CH-1015 Lausanne, Switzerland

${ }^{d}$ Institute for Nuclear Research of the Russian Academy of Sciences, 60th October Anniversary Prospect, 7a, 117312 Moscow, Russia

${ }^{e}$ Physikalisches Institut, Albert-Ludwigs-Universität Freiburg,

Hermann-Herder-Strasse 3, 79104 Freiburg, Germany

E-mail: barvin@td.lpi.ru, blas.diego@gmail.com, mario.herrerovalea@epfl.ch, Sergey.Sibiryakov@cern.ch, christian.steinwachs@physik.uni-freiburg.de

ABSTRACT: Using the background-field method we demonstrate the Becchi-Rouet-StoraTyutin (BRST) structure of counterterms in a broad class of gauge theories. Put simply, we show that gauge invariance is preserved by renormalization in local gauge field theories whenever they admit a sensible background-field formulation and anomaly-free path integral measure. This class encompasses Yang-Mills theories (with possibly Abelian subgroups) and relativistic gravity, including both renormalizable and non-renormalizable (effective) theories. Our results also hold for non-relativistic models such as Yang-Mills theories with anisotropic scaling or Hořava gravity. They strengthen and generalize the existing results in the literature concerning the renormalization of gauge systems. Locality of the BRST construction is emphasized throughout the derivation. We illustrate our general approach with several explicit examples.

Keywords: BRST Quantization, Effective Field Theories, Gauge Symmetry, Models of Quantum Gravity

ARXIV EPRINT: 1705.03480 


\section{Contents}

1 Introduction 1

2 Assumptions and proposition 5

2.1 Gauge algebra 5

$\begin{array}{ll}2.2 \text { Background gauge } & 7\end{array}$

2.3 Absence of anomalies and locality of divergences 9

$\begin{array}{ll}2.4 \text { Proposition: BRST structure of the renormalized action } & 10\end{array}$

3 BRST structure for selected gauge theories $\quad \mathbf{1 2}$

$\begin{array}{lll}3.1 & \text { Relativistic Yang-Mills in }(3+1) \text { dimensions } & 12\end{array}$

3.2 Higher-derivative relativistic gravity in $(3+1)$ dimensions 13

$\begin{array}{lll}3.3 & \text { Projectable Hořava gravity in }(d+1) \text { dimensions } & 15\end{array}$

$\begin{array}{lll}3.4 & \text { General relativity as effective field theory in }(3+1) \text { dimensions } & 18\end{array}$

$\begin{array}{lll}4 & \text { Equations for the effective action } & 18\end{array}$

5 Structure of divergences $\quad \mathbf{2 0}$

5.1 Separating the background field dependence 21

$\begin{array}{ll}5.2 & \text { Ghost-dependent contribution } \\ 5.3 & 23\end{array}$

$\begin{array}{lll}5.3 & \text { Subtraction and field redefinition } & 25\end{array}$

6 Counterterms and nonlinear field renormalization in $O(N)$ model: ex$\begin{array}{lr}\text { plicit one-loop calculation } & 27\end{array}$

7 Conclusions and discussion $\quad 32$

A Derivation of Slavnov-Taylor and Ward identities 34

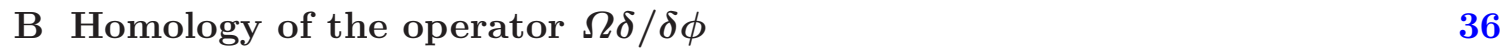

C Quadratic form for perturbations in the $O(N)$ model $\quad 37$

\section{Introduction}

A central question in the perturbative quantization of gauge field theories is to what extent the gauge symmetry is preserved by renormalization. Intuition tells that in the absence of anomalies, i.e. when the measure in the path integral is gauge invariant, the counterterms required to cancel the ultraviolet divergences should be gauge invariant as well. A rigorous proof of this assertion, however, is highly non-trivial due to the breaking of the gauge 
symmetry required to quantize gauge theories (gauge-fixing procedure). The original gauge invariance still survives in the Becchi-Rouet-Stora-Tyutin (BRST) [1-3] structure of the gauge-fixed action $\Sigma$ which remains invariant under infinitesimal variations generated by the nilpotent BRST operator. At tree level, $\Sigma$ is a sum of a BRST exact part responsible for the gauge fixing and the classical gauge invariant action which depends only on the physical fields (gauge fields and matter) and is independent of the Faddeev-Popov ghosts. The physical content of gauge invariance will be retained if this BRST structure persists under renormalization. In particular, it will guarantee that the partition function obeys Slavnov-Taylor identities at all orders of the perturbative expansion.

In addition, to preserve the key properties of quantum field theory, the BRST structure must be compatible with locality. Namely, starting from a gauge theory with a local ${ }^{1}$ Lagrangian, both the BRST-exact and the gauge-invariant parts of the renormalized action must be given by integrals of local Lagrange densities.

In the textbook examples of renormalizable relativistic theories, such as quantum electrodynamics or Yang-Mills (YM) theory, the previous properties can be proven by "brute force": one first writes down all possible counterterms allowed by power counting and then solves the equations for their coefficients following from the Slavnov-Taylor identities. The last step required to bring the renormalized action into the BRST form is a field redefinition. Positive canonical dimensions of the fields and the absence of any coupling constants with negative dimensionality imply in these simple cases that the field redefinition must have the form of a multiplicative wavefunction renormalization, whose coefficient is easy to find, see e.g. [4].

In general the situation is much more involved. This is the case, for example, in non-renormalizable theories (understood as effective field theories, EFTs) where one encounters coupling constants of negative dimension. In these cases an explicit solution of the Slavnov-Taylor identities appears infeasible. Even if such solution were available, the field redefinition bringing it to the BRST form could be nonlinear and arbitrarily complicated, rendering a brute-force search for it hopeless. The same is true for renormalizable higher-derivative gravity [5] where the canonical dimension of the metric is zero, implying that its renormalization can be, and actually is, nonlinear. To study the consistency of the BRST structure with renormalization in this type of theories one needs more powerful methods.

It is well-known that the classification of possible counterterms arising in general gauge theories requires computing the cohomology of an extended BRST operator [6-10] (see also [4]). To be compatible with the BRST structure, the latter must consist of local ${ }^{2}$ gauge-invariant functionals of physical fields only. This was indeed demonstrated in [11-13] for the EFT consisting of general relativity coupled to YM with semisimple gauge group extended by arbitrary gauge invariant higher-order operators. These references use the

\footnotetext{
${ }^{1}$ That is represented as a sum of terms depending on fields and their derivatives at a point. This sum can, in principle, be infinite provided terms with higher number of derivatives are treated perturbatively, as it happens in effective field theories.

${ }^{2}$ The requirement of locality is crucial. Refs. [7-9] studying the BRST cohomology in general gauge theories do not guarantee its locality and have to postulate it as an additional assumption.
} 
advanced mathematical apparatus of local cohomology theory. Notably, for gauge groups with Abelian factors they still leave room for non-trivial cohomologies different from gauge invariant functionals which, if generated by divergences, would imply deformations of the original gauge symmetry. Additional arguments must be invoked to forbid the appearance of such counterterms in the studied cases [10].

The purpose of our work is to address the BRST structure of renormalized actions in general gauge field theories admitting background field gauges. Our motivation is twofold. First, we will provide a new, and we believe simpler, derivation of the results concerning the renormalization of Einstein-YM theories and strengthen them for the case of theories with Abelian subgroups. Second, our analysis covers a broader class of gauge theories not considered in the classic papers [11-13]. This includes, in particular, the higher-derivative gravity and gauge/gravity theories without relativistic invariance.

Non-relativistic gauge theories play a prominent role in condensed matter physics [14-17] (see also references therein), investigations of non-relativistic Weyl invariance and holography [18, 19], and may be relevant for particle model building [20-22] (see [23] for a summary of extra motivations and results in non-relativistic gauge theories). Furthermore, abandoning relativistic invariance (while keeping the gauge group of timedependent spatial diffeomorphisms) allows one to construct power-counting renormalizable models of gravity in arbitrary spacetime dimensions including the phenomenologically interesting case of $(3+1)$ dimensions $[24,25]$. The renormalizability beyond power counting was established in [26] for a large subset of these gravity models, the so-called projectable Hořava gravities. It was assumed in [26] that renormalization preserves gauge invariance, which was explicitly demonstrated only at one loop. One of the goals of the present paper is to demonstrate the validity of this assumption to all loop orders and thereby complete the proof of renormalizability of projectable Hořava gravity.

Our approach is based on the background field method [27-29] (see also [30, 31]), a powerful tool for calculating the quantum effective action in gauge theories and gravity. The main virtue of this method is that it preserves the gauge invariance of the calculations even after gauge fixing. This is achieved by the introduction of additional external sources - background fields - in such a way that the partition function remains invariant under simultaneous gauge transformations of the variables in the path integral ("quantum fields") and the background fields. We denote this transformation "background-gauge transformation". At the same time the quantum gauge transformations acting only on the quantum fields are broken by gauge fixing and the path integral is well defined (at least perturbatively). The construction of background-covariant gauge fixing conditions is straightforward in theories containing fields in linear representations of gauge groups with linear generators. These conditions imply that the background-gauge symmetry is preserved by renormalization which serves as a strong selection criterion for possible counterterms. This method greatly simplifies the renormalization of coupling constants in the one-loop approximation after the background fields are identified with the mean value of the quantum fields [32-34]. In this case the counterterms take a manifestly gauge invariant form.

Beyond one-loop the situation becomes more complicated. The subtraction of subdivergences necessary to eliminate the nonlocal infinities requires counterterms where the quantum fields are distinct from the background fields. Background-gauge invariance is 
not sufficient to completely fix the structure of such counterterms and the BRST structure associated to the quantum gauge transformations must be exploited, as is done in the cases of gauges without background fields [35-39]. In practical calculations these counterterms can sometimes be avoided by subtle methods that have been developed for YM and relativistic gravity. However, these techniques generically feature nonlocal divergences at intermediate steps of the calculations, that cancel only in the final quantities evaluated on-shell [40-44]. The presence of nonlocal divergences makes these methods inappropriate for a general analysis of renormalizability. More recently it has been advocated [45] that the use of a background gauge combined with the standard subtraction scheme provides a valuable tool for such analysis (see also [46, 47]). This reference uses the Batalin-Vilkovisky formalism [48-51] to prove the existence of a canonical transformation bringing the renormalized action to the BRST form. However, this requires introducing background field counterparts for all quantum fields of the theory including Faddeev-Popov ghosts and, moreover, the addition of Batalin-Vilkovisky antifields for all background fields. Such proliferation of objects makes the construction rather baroque and obscures the subtleties of the derivation.

In this paper we adopt a different strategy and proceed along the lines of traditional cohomology analysis. Our key finding is that the background-gauge invariance greatly facilitates the computation of the local BRST cohomology. The latter reduces to cohomologies of a few simpler nilpotent operators that are readily computed using elementary algebraic techniques. The resulting constraints on the form of the renormalized action imply that, upon an appropriate field redefinition, it acquires the desired BRST form (a local gauge-invariant functional plus a BRST-exact piece). The argument does not involve any power-counting considerations. When available, such considerations lead to further refinements which we discuss. We keep track of locality at all steps of the derivation.

Our proof applies to theories characterized by the following properties: the gauge algebra is irreducible and closes off-shell; the gauge generators depend on the fields at most linearly; the structure functions are field independent. These conditions ensure that the theory admits a convenient background-covariant gauge fixing. Additionally, we assume the absence of anomalies and locality of the leading ultraviolet divergences (ones that remain after subtraction of subdivergences). The latter requirement should not be confused with locality of the BRST decomposition, which is not postulated a priori, but is derived from the previous assumptions.

The above class is quite broad. It encompasses renormalizable and non-renormalizable (effective) theories with Abelian and non-Abelian gauge groups, general relativity and higher-derivative gravity. In addition to the standard relativistic versions of these theories, it also includes their non-relativistic generalizations [24, 25, 52-54]. As a corollary of our general result we establish for the first time the compatibility of the BRST structure with renormalization in projectable Hořava gravity [24, 25] which completes the proof of its renormalizability. A notable example that is not covered by our study is supergravity where the gauge algebra closes only on-shell. ${ }^{3}$

\footnotetext{
${ }^{3}$ For $N=1$ supergravity in four spacetime dimensions, the off-shell closure of the algebra can be achieved by introduction of auxiliary fields, but then the generators become nonlinear in the fields [55].
} 
While various ingredients of our analysis have already appeared in the literature, to the best of our knowledge, they have never been put together. To make the presentation self-contained we review these ingredients in the relevant sections. Several concrete examples aim to illustrate the physical content of the general result. For simplicity we focus throughout the paper on theories with bosonic gauge parameters.

The paper is organized as follows. In section 2 we describe our assumptions, introduce the background gauge fixing and formulate our main result (section 2.4). In section 3 we illustrate its implications on several examples. We discuss explicitly the standard renormalizable YM in $(3+1)$ dimensions, relativistic higher-derivative gravity in $(3+1)$ dimensions, projectable Hořava gravity in general dimensions and general relativity in $(3+1)$ dimensions (understood as an effective theory). In section 4 we turn to the proof of our general result and derive the equations satisfied by the effective action as a consequence of the background and quantum gauge invariances. These equations are used to analyze the structure of the divergent counterterms in section 5, which is the central part of the paper. Here we formulate the cohomology problem and use the background-gauge invariance to split it into several subproblems. Solving them we fix the structure of the renormalized action and demonstrate existence of a field redefinition that casts it into the BRST form advocated in section 2.4. This completes the formal proof. Section 6 is devoted to one more example - the $O(N)$ vector model in $(1+1)$ spacetime dimensions written as an Abelian gauge theory. This example is interesting as it features nonlinear wavefunction renormalization, being at the same time simple enough to admit an explicit treatment. We verify at one loop that the counterterms in this theory have the structure determined by the general argument. We conclude in section 7. Appendix A contains the derivation of the SlavnovTaylor and Ward identities for the partition function. In appendix B we prove a lemma about the cohomology of an operator appearing in our analysis. Some formulae used in the computation of the effective action of the $O(N)$ model are summarized in appendix $\mathrm{C}$.

\section{Assumptions and proposition}

\subsection{Gauge algebra}

We consider a theory with local gauge and matter fields $\varphi^{a}$, where $a$ is a collective notation for all indices and the coordinates. The theory is described by the action $S[\varphi]$ which is an integral of a local Lagrangian density $\mathcal{L}(\varphi)$. The latter is expanded as a sum of terms depending on the fields $\varphi^{a}$ and their finite-order derivatives at a given point. ${ }^{4}$ The action $S[\varphi]$ is invariant under gauge transformations with local bosonic parameters $\varepsilon^{\alpha}$. The transformations are assumed to have at most linear dependence on the fields,

$$
\delta_{\varepsilon} \varphi^{a}=R_{\alpha}^{a}(\varphi) \varepsilon^{\alpha}, \quad R_{\alpha}^{a}(\varphi)=P_{\alpha}^{a}+R_{b \alpha}^{a} \varphi^{b}, \quad R_{\alpha}^{a}(\varphi) \frac{\delta S[\varphi]}{\delta \varphi^{a}}=0
$$

\footnotetext{
${ }^{4}$ Throughout the text the dependence of local functions on the fields and their finite-order derivatives will be denoted by round brackets, while square brackets will denote the functional dependence of integral quantities with local or nonlocal integrands.
} 
We further assume that the gauge algebra closes off-shell,

$$
\left[\delta_{\varepsilon}, \delta_{\eta}\right] \varphi^{a}=\delta_{\varsigma} \varphi^{a}
$$

where

$$
\varsigma^{\alpha}=C_{\beta \gamma}^{\alpha} \varepsilon^{\beta} \eta^{\gamma}
$$

and $C_{\beta \gamma}^{\alpha}$ are field-independent structure functions. The closure condition implies the relations,

$$
\begin{aligned}
R_{b \alpha}^{a} P_{\beta}^{b}-R_{b \beta}^{a} P_{\alpha}^{b} & =P_{\gamma}^{a} C_{\alpha \beta}^{\gamma}, \\
R_{b \alpha}^{a} R_{c \beta}^{b}-R_{b \beta}^{a} R_{c \alpha}^{b} & =R_{c \gamma}^{a} C_{\alpha \beta}^{\gamma} .
\end{aligned}
$$

In addition, $C_{\beta \gamma}^{\alpha}$ obey the Jacobi identities,

$$
C_{\beta[\gamma}^{\alpha} C_{\lambda \mu]}^{\beta}=0
$$

where the square brackets mean anisymmetrization over the respective indices.

Next, we require that the set of gauge generators $R_{\alpha}^{a}(\varphi)$ is locally complete and irreducible. These properties are defined as follows:

(i) Local completeness $[51,56]$ : any local operator $X_{\alpha}^{a}(\varphi)$ satisfying the equation

$$
\frac{\delta S}{\delta \varphi^{a}} X_{\alpha}^{a}=0
$$

is represented as a linear combination of the gauge generators and equations of motion,

$$
X_{\alpha}^{a}=R_{\beta}^{a} Y_{\alpha}^{\beta}+\frac{\delta S}{\delta \varphi^{b}} I_{\alpha}^{[b a]},
$$

where $Y_{\alpha}^{\beta}$ and $I_{\alpha}^{[b a]}$ are local and $I_{\alpha}^{[b a]}$ is antisymmetric in its indices. The locality condition means that $Y_{\alpha}^{\beta}$ and $I_{\alpha}^{[b a]}$ are non-zero only if the coordinates corresponding to $\beta$ and $\alpha$ or $a, b$ and $\alpha$ coincide.

(i) Irreducibility [48-50]: let $\varphi_{0}^{a}$ be a solution of the equations of motion, so that

$$
\frac{\delta S}{\delta \varphi^{a}}\left(\varphi_{0}\right)=0
$$

If a gauge parameter $\varepsilon^{\alpha}$ satisfies the relations

$$
R_{\alpha}^{a}\left(\varphi_{0}\right) \varepsilon^{\alpha}=0
$$

then $\varepsilon^{\alpha}=0$. In other words, gauge transformations act non-trivially on on-shell configurations. 
The class of theories described above is quite broad. It includes, in particular, relativistic Abelian and non-Abelian gauge theories together with their extensions by higherderivative operators, general relativity and relativistic higher-derivative gravity, e.g. [5]. Moreover, it contains non-relativistic generalizations of these theories. Some examples are discussed in section 3 and in section 6 . As we mentioned, a notable exception from this class is supergravity, both due to the fermionic nature of the gauge parameter and openness of the gauge algebra.

For the sake of clarity, we focus in what follows on theories where all fields $\varphi^{a}$ are bosonic. The inclusion of fermionic matter fields is straightforward, but would complicate the formulae by additional $(-1)$ factors.

\subsection{Background gauge}

To quantize the theory we need to fix the gauge. We introduce the background fields ${ }^{5} \phi^{a}$ and choose the gauge fixing function $\chi^{\alpha}(\varphi, \phi)$ in such a way that it transforms covariantly under simultaneous local gauge transformation of $\varphi^{a}$ and $\phi^{a}$ with the same parameter $\varepsilon$ but their own generators $R_{\alpha}^{a}(\varphi)$ and $R_{\alpha}^{a}(\phi)$ respectively,

$$
\delta_{\varepsilon} \varphi^{a}=R_{\alpha}^{a}(\varphi) \varepsilon^{\alpha}, \quad \delta_{\varepsilon} \phi^{a}=R_{\alpha}^{a}(\phi) \varepsilon^{\alpha} .
$$

Covariance of $\chi^{\alpha}$ under the transformations (2.10) implies,

$$
\delta_{\varepsilon} \chi^{\alpha} \equiv \frac{\delta \chi^{\alpha}}{\delta \varphi^{a}} \delta_{\varepsilon} \varphi^{a}+\frac{\delta \chi^{\alpha}}{\delta \phi^{a}} \delta_{\varepsilon} \phi^{a}=-C_{\beta \gamma}^{\alpha} \chi^{\beta} \varepsilon^{\gamma} .
$$

We will refer to (2.10) as "background-gauge transformations" and to $\chi^{\alpha}(\varphi, \phi)$ as "background-covariant gauge conditions". We further choose $\chi^{\alpha}$ to be linear in the difference $\left(\varphi^{a}-\phi^{a}\right)$,

$$
\chi^{\alpha}(\varphi, \phi)=\chi_{a}^{\alpha}(\phi)\left(\varphi^{a}-\phi^{a}\right) .
$$

The gauge-fixing function is assumed to be local in space-time, i.e. it depends only on the values of the fields and their derivatives of finite order at a point.

The gauge fixing is implemented by the BRST procedure [1-3] (see also [4]). Labelling anticommuting ghosts $\omega^{\alpha}$, antighosts $\bar{\omega}_{\alpha}$ and the Lagrange multiplier $b_{\alpha}$ with the condensed gauge index $\alpha$, we define the standard action of the BRST operator $s$

$$
\begin{aligned}
s \varphi^{a} & =R_{\alpha}^{a}(\varphi) \omega^{\alpha}, \\
s \omega^{\alpha} & =\frac{1}{2} C_{\beta \gamma}^{\alpha} \omega^{\beta} \omega^{\gamma}, \\
s \bar{\omega}_{\alpha} & =b_{\alpha}, \\
s b_{\alpha} & =0 .
\end{aligned}
$$

The closure conditions (2.4), (2.5) imply that $s$ is nilpotent. The background fields $\phi^{a}$ are invariant under the action of $s$. Next, we introduce two sets of anticommuting auxiliary fields $\gamma_{a}, \Omega^{a}$ and a commuting field $\zeta_{\alpha}$. They are also invariant under the BRST

\footnotetext{
${ }^{5}$ For bosonic gauge algebras that we consider in this paper, it is sufficient to introduce background counterparts to bosonic fields only, even if the theory contains fermionic matter.
} 
transformations generated by $\boldsymbol{s}$. We define the gauge fermion as

$$
\Psi_{0}[\varphi, \omega, \bar{\omega}, b, \phi, \gamma, \zeta]=\bar{\omega}_{\alpha}\left(\chi_{a}^{\alpha}(\phi)\left(\varphi^{a}-\phi^{a}\right)-\frac{1}{2} O^{\alpha \beta}(\phi) b_{\beta}\right)-\gamma_{a}\left(\varphi^{a}-\phi^{a}\right)+\zeta_{\alpha} \omega^{\alpha}
$$

Here $O^{\alpha \beta}(\phi)$ is an invertible local operator that can, in general, depend on the background fields ${ }^{6}$ and transforms covariantly under the background-gauge transformations. Finally, we construct the gauge-fixed action,

$$
\Sigma_{0}[\varphi, \omega, \bar{\omega}, b, \phi, \gamma, \zeta, \Omega]=S[\varphi]+\boldsymbol{Q} \Psi_{0},
$$

with

$$
\boldsymbol{Q}=\boldsymbol{s}+\Omega^{a} \frac{\delta}{\delta \phi^{a}} .
$$

Following [35-39] we have extended the usual BRST operator in such a way that it controls not only the field BRST transformations but also the variation of the gauge-fixing term under the changes of $\phi$. Clearly, $\boldsymbol{Q}$ is nilpotent due to the anticommuting nature of $\Omega^{a}$. Explicitly, the action (2.15) reads,

$$
\begin{aligned}
& \Sigma_{0}[\varphi, \omega, \bar{\omega}, b, \phi, \gamma, \zeta, \Omega]=S[\varphi]+b_{\alpha} \chi_{a}^{\alpha}(\phi)\left(\varphi^{a}-\phi^{a}\right)-\frac{1}{2} O^{\alpha \beta}(\phi) b_{\alpha} b_{\beta}-\bar{\omega}_{\alpha} \chi_{a}^{\alpha}(\phi) R_{\beta}^{a}(\varphi) \omega^{\beta} \\
& +\gamma_{a} R_{\alpha}^{a}(\varphi) \omega^{\alpha}+\frac{1}{2} \zeta_{\alpha} C_{\beta \gamma}^{\alpha} \omega^{\beta} \omega^{\gamma}+\Omega^{c} \bar{\omega}_{\alpha}\left[\frac{\delta \chi_{b}^{\alpha}}{\delta \phi^{c}}(\varphi-\phi)^{b}-\chi_{c}^{\alpha}-\frac{1}{2} \frac{\delta O^{\alpha \beta}}{\delta \phi^{c}} b_{\beta}\right]+\Omega^{c} \gamma_{c}
\end{aligned}
$$

One recognizes the gauge fixing part (second and third terms in the first line) ${ }^{7}$ and the Faddeev-Popov action for the ghost-antighost pair (last term in the first line). The second line collects the dependence on the auxiliary fields $\gamma_{a}, \zeta_{\alpha}$ and $\Omega^{a}$. Notice that $\gamma_{a}$ and $\zeta_{\alpha}$ couple as sources to the BRST variations of $\varphi^{a}$ and $\omega^{\alpha}$ respectively.

In view of the nilpotency of $\boldsymbol{Q}$ the gauge-fixed action is BRST-invariant,

$$
Q \Sigma_{0}=0
$$

This equation will be used below to derive the Slavnov-Taylor identities constraining the ultraviolet divergences. Also, for background-covariant gauges of the above type, $\Psi_{0}$ and $\Sigma_{0}$ have an additional symmetry: they are invariant under background-gauge transformations (2.10),

$$
\delta_{\varepsilon} \Psi_{0}=0, \quad \delta_{\varepsilon} \Sigma_{0}=0,
$$

if simultaneously with $\varphi^{a}$ and $\phi^{a}$ we transform all fields in the appropriate linear representations:

$$
\delta_{\varepsilon} \gamma_{a}=-\gamma_{b} R_{a \alpha}^{b} \varepsilon^{\alpha}, \quad \delta_{\varepsilon} \omega^{\alpha}=-C_{\beta \gamma}^{\alpha} \omega^{\beta} \varepsilon^{\gamma}, \quad \delta_{\varepsilon} \zeta_{\alpha}=\zeta_{\beta} C_{\alpha \gamma}^{\beta} \varepsilon^{\gamma}, \quad \delta_{\varepsilon} \Omega^{\alpha}=R_{b \alpha}^{a} \Omega^{b} \varepsilon^{\alpha},
$$

\footnotetext{
${ }^{6}$ This dependence is, in fact, inevitable in gravity (see section 3).

${ }^{7}$ Gaussian integration over the Lagrange multiplier $b_{\alpha}$ gives a familiar gauge breaking term $\frac{1}{2} \chi^{\alpha} O_{\alpha \beta}^{-1} \chi^{\beta}$ with the weighting factor inverse to $O^{\alpha \beta}$.
} 
and similarly for $\bar{\omega}_{\alpha}$ and $b_{\alpha}$. Note that for theories with diffeomorphism invariance $\omega^{\alpha}$ transforms as a contravariant vector, whereas $\bar{\omega}_{\alpha}, b_{\alpha}, \gamma_{a}, \zeta_{\alpha}$ are vector/tensor densities. Finally, the action (2.17) possesses a global U(1) symmetry corresponding to the ghost number with the following assignment of charges:

$$
\begin{array}{lll}
\operatorname{gh}(\varphi)=\operatorname{gh}(\phi)=\operatorname{gh}(b)=0, & & \operatorname{gh}(\omega)=\operatorname{gh}(\Omega)=+1, \\
\operatorname{gh}(\bar{\omega})=\operatorname{gh}(\gamma)=-1, & & \operatorname{gh}(\zeta)=-2 .
\end{array}
$$

Using (2.17) as the tree-level action and introducing sources coupled to the "quantum" fields $(\varphi, \omega, \bar{\omega}, b)$ we write the "bare" generating functional for connected graphs,

$$
W_{0}[J, \bar{\xi}, \xi, y, \phi, \gamma, \zeta, \Omega]=-\hbar \log \int d \Phi \exp \left[-\frac{1}{\hbar}\left(\Sigma_{0}+J_{a}\left(\varphi^{a}-\phi^{a}\right)+\bar{\xi}_{\alpha} \omega^{\alpha}+\xi^{\alpha} \bar{\omega}_{\alpha}+y^{\alpha} b_{\alpha}\right)\right] .
$$

Here we have collectively denoted all quantum fields by $\Phi$ in the integration measure and explicitly included the Planck constant $\hbar$ as a counting parameter for the order of the loop expansion. ${ }^{8}$

\subsection{Absence of anomalies and locality of divergences}

We impose two more conditions on the theory. First, we postulate the absence of gauge anomalies, i.e. the existence of a regularization prescription that preserves the gauge invariance of the functional integration measure. This is achieved by dimensional regularization in many cases.

Second, we require that a variant of the standard subtraction scheme (e.g. minimal subtraction) [57] eliminates all nonlocal divergences. Let us expand on this point. In the standard scheme the counterterms are constructed inductively in the number of loops $L$ or, equivalently, in the powers of $\hbar$. Let us assume that at order $\mathcal{O}\left(\hbar^{L-1}\right)$ we have already constructed the renormalized action

$$
\Sigma_{L-1}=\Sigma_{0}+\sum_{l=1}^{L-1} \hbar^{l} \Sigma_{l}^{C},
$$

where $\Sigma_{0}$ is the tree-level action $(2.17)$ and $\Sigma_{l}^{C}$ are divergent local counterterms. This action is such that the generating functional $W_{L-1}$ defined by the formula analogous to (2.22) with the replacement $\Sigma_{0} \mapsto \Sigma_{L-1}$ produces Green's functions that are finite at $(L-1)$ loops.

Next, we introduce the mean fields ${ }^{9}$ as functional derivatives of the generating functional with respect to the sources, ${ }^{10}$

$$
\left\langle\varphi^{a}\right\rangle-\phi^{a}=\frac{\delta W}{\delta J_{a}}, \quad\left\langle\omega^{\alpha}\right\rangle=\frac{\delta W}{\delta \bar{\xi}_{\alpha}}, \quad\left\langle\bar{\omega}_{\alpha}\right\rangle=\frac{\delta W}{\delta \xi^{\alpha}}, \quad\left\langle b_{\alpha}\right\rangle=\frac{\delta W}{\delta y^{\alpha}},
$$

\footnotetext{
${ }^{8}$ Throughout the paper we work with Euclidean field theory and use the corresponding definition of the generating functional. As the operator $O^{\alpha \beta}$ in (2.17) is usually chosen positive-definite, the convergence of the path integral requires that the integration in $b_{\alpha}$ runs along the imaginary axis. This subtlety does not affect our analysis.

${ }^{9}$ These should not be confused with the background fields $\phi^{a}$.

${ }^{10}$ We fix the sign of the derivatives with respect to the anticommuting variables by placing the differential on the left, $d f=d \theta f^{\prime}(\theta)$.
} 
and define the effective action $\Gamma$ as the Legendre transform of $W$,

$$
\Gamma[\langle\varphi\rangle,\langle\omega\rangle,\langle\bar{\omega}\rangle,\langle b\rangle, \phi, \gamma, \zeta, \Omega]=W-J_{a}\left(\left\langle\varphi^{a}\right\rangle-\phi^{a}\right)-\bar{\xi}_{\alpha}\left\langle\omega^{\alpha}\right\rangle-\xi^{\alpha}\left\langle\bar{\omega}_{\alpha}\right\rangle-y^{\alpha}\left\langle b_{\alpha}\right\rangle .
$$

Clearly, it satisfies,

$$
\frac{\delta \Gamma}{\delta\left\langle\varphi^{a}\right\rangle}=-J_{a}, \quad \frac{\delta \Gamma}{\delta\left\langle\omega^{\alpha}\right\rangle}=\bar{\xi}_{\alpha}, \quad \frac{\delta \Gamma}{\delta\left\langle\bar{\omega}_{\alpha}\right\rangle}=\xi^{\alpha}, \quad \frac{\delta \Gamma}{\delta\left\langle b_{\alpha}\right\rangle}=-y^{\alpha},
$$

The $(L-1)$-th order effective action has the form

$$
\Gamma_{L-1}=\Sigma_{0}+\sum_{l=1}^{\infty} \hbar^{l} \Gamma_{L-1}^{(l)}
$$

where $\Gamma_{L-1}^{(l)}$ is the contribution of diagrams with $l$ loops. By the assumption of the induction step, all terms $\Gamma_{L-1}^{(l)}$ with $l \leq L-1$ are finite and the divergence of the $L$-th term,

$$
\Gamma_{L-1, \infty}^{(L)} \equiv \Gamma_{L, \infty}[\langle\varphi\rangle,\langle\omega\rangle,\langle\bar{\omega}\rangle,\langle b\rangle, \phi, \gamma, \zeta, \Omega]
$$

is local. Then the counterterm $\Sigma_{L}^{C}$ is identified with $-\Gamma_{L, \infty}$ where the mean fields are replaced by the quantum fields,

$$
\Sigma_{L}[\varphi, \omega, \bar{\omega}, b, \phi, \gamma, \zeta, \Omega]=\Sigma_{L-1}-\hbar^{L} \Gamma_{L, \infty}[\varphi, \omega, \bar{\omega}, b, \phi, \gamma, \zeta, \Omega] .
$$

According to the standard theorems [57] (see [58] for the generalization to theories without Lorentz invariance), this subtraction removes the $L$-loop divergences, as well as all subdivergences in $(L+1)$-loop diagrams.

In relativistic gauge theories with Lorentz-covariant gauge fixing, this guarantees that the remaining divergence of order $\mathcal{O}\left(\hbar^{L+1}\right)$ in the effective action $\Gamma_{L}$ is local and the subtraction can be repeated at the $(L+1)$-th loop order. The situation is less straightforward in the absence of Lorentz invariance [26] and the locality of the remaining divergences must be verified in every given theory. It was shown to hold for non-relativistic YM theories with anisotropic (Lifshitz) scaling and projectable Hořava gravity [26]. In the present paper we postulate it as a property of the class of theories under study.

To avoid cluttered notations, we will omit the averaging symbols on the arguments of the effective action $\Gamma$ in what follows.

\subsection{Proposition: BRST structure of the renormalized action}

We will show that a slight modification of the subtraction prescription by the inclusion of additional local terms of order $\mathcal{O}\left(\hbar^{L+1}\right)$ on the r.h.s. of (2.29) leads to a renormalized action $\Sigma_{L}$ that preserves the BRST structure. More precisely, our result is formulated as follows.

Let us denote the fields coupled to the external sources $J, \bar{\xi}$ by $\tilde{\varphi}, \tilde{\omega}$ and consider local field reparameterizations of the form,

$$
\tilde{\varphi}^{a}=\tilde{\varphi}_{L}^{a}(\varphi, \omega, \phi, \hat{\gamma}, \zeta, \Omega) \quad \tilde{\omega}^{\alpha}=\tilde{\omega}_{L}^{\alpha}(\varphi, \omega, \phi, \hat{\gamma}, \zeta, \Omega)
$$


where we have introduced the combination

$$
\hat{\gamma}_{a}=\gamma_{a}-\bar{\omega}_{\alpha} \chi_{a}^{\alpha}(\phi)
$$

that will play an important role below. Upon the field redefinition the $L$-th order generating functional reads, ${ }^{11}$

$$
W_{L}[J, \bar{\xi}, \xi, y, \phi, \gamma, \zeta, \Omega]=-\hbar \log \int d \Phi \exp \left[-\frac{1}{\hbar}\left(\Sigma_{L}+J_{a}\left(\tilde{\varphi}_{L}^{a}-\phi^{a}\right)+\bar{\xi}_{\alpha} \tilde{\omega}_{L}^{\alpha}+\xi^{\alpha} \bar{\omega}_{\alpha}+y^{\alpha} b_{\alpha}\right)\right] .
$$

We will demonstrate the existence of a field redefinition (2.30) such that $\Sigma_{L}$ takes the form,

$$
\Sigma_{L}[\varphi, \omega, \bar{\omega}, b, \phi, \gamma, \zeta, \Omega]=S_{L}[\varphi]+\boldsymbol{Q} \Psi_{L}[\varphi, \omega, \bar{\omega}, b, \phi, \gamma, \zeta, \Omega],
$$

where $S_{L}[\varphi]$ is a gauge invariant local functional and the BRST operator $\boldsymbol{Q}$ has been defined in (2.16). The gauge fermion $\Psi_{L}$ is a local functional with ghost number $(-1)$ which is invariant under background-gauge transformations (2.10), (2.20) and has the form,

$$
\Psi_{L}=\hat{\Psi}_{L}[\varphi, \omega, \phi, \hat{\gamma}, \zeta, \Omega]-\frac{1}{2} \bar{\omega}_{\alpha} O^{\alpha \beta}(\phi) b_{\beta},
$$

where

$$
\hat{\Psi}_{L}=-\hat{\gamma}_{a}\left(\varphi^{a}-\phi^{a}\right)+\zeta_{\alpha} \omega^{\alpha}+\mathcal{O}(\hbar) .
$$

Further, the reparameterization (2.30) itself is generated by the gauge fermion,

$$
\tilde{\varphi}_{L}^{a}=\phi^{a}-\frac{\delta \Psi_{L}}{\delta \gamma_{a}}, \quad \tilde{\omega}_{L}^{\alpha}=\frac{\delta \Psi_{L}}{\delta \zeta_{\alpha}} .
$$

Together with (2.35) this implies that at tree level $\tilde{\varphi}, \tilde{\omega}$ coincide with $\varphi, \omega$ and the gauge fermion $\Psi_{L}$ coincides with the expression (2.14). Thus, we recover (2.22) at tree level.

Eqs. (2.32)-(2.36) represent a generalization of the construction described in section 2.2 that is forced on us by renormalization. Notice that the sources $J_{a}, \bar{\xi}_{\alpha}$ now couple to composite local operators that in general depend not only on the quantum fields, but also on the external backgrounds $\phi^{a}, \gamma_{a}, \zeta_{\alpha}, \Omega^{a}$. Nevertheless, this is not problematic due to the property $(2.35),(2.36)$ that ensures linearity of the coupling at leading order in $\hbar$.

We will see in section 3 and section 6 that in many interesting theories, that are typically renormalizable, power counting considerations strongly restrict the dependence of the renormalized gauge fermion $\hat{\Psi}_{L}$ on the auxiliary fields. Namely, in these cases $\hat{\Psi}_{L}$ is independent of $\Omega^{a}$ and can depend on $\hat{\gamma}_{a}, \zeta_{\alpha}$ only linearly,

$$
\hat{\Psi}_{L}=-\hat{\gamma}_{a} U_{L}^{a}(\varphi, \phi)+\zeta_{\alpha} \omega^{\beta} V_{L \beta}^{\alpha}(\varphi, \phi),
$$

with

$$
\begin{aligned}
& U_{L}^{a}=\varphi^{a}-\phi^{a}+\sum_{l=1}^{L} \hbar^{l} \boldsymbol{u}_{l}^{a}(\varphi, \phi), \\
& V_{L \beta}^{\alpha}=\delta_{\beta}^{\alpha}+\sum_{l=1}^{L} \hbar^{l} \boldsymbol{v}_{l \beta}^{\alpha}(\varphi, \phi) .
\end{aligned}
$$

\footnotetext{
${ }^{11}$ We disregard the functional Jacobian $|\delta \tilde{\Phi} / \delta \Phi|$ which gives a contribution to the Lagrangian of the form $\sum_{n} \mathcal{O}_{n} \partial \ldots \partial \delta(0)$ with some local operators $\mathcal{O}_{n}$. Such contributions vanish in dimensional regularization.
} 
Correspondingly, the field redefinition (2.36) bringing the counterterms into the BRSTinvariant form simplifies to

$$
\tilde{\varphi}_{L}^{a}=\phi^{a}+U_{L}^{a}(\varphi, \phi), \quad \tilde{\omega}_{L}^{\alpha}=V_{L \beta}^{\alpha}(\varphi, \phi) \omega^{\beta}
$$

In this case it does not involve the auxiliary sources $\gamma_{a}, \zeta_{\alpha}, \Omega^{a}$.

\section{BRST structure for selected gauge theories}

In this section we illustrate the notions and results described above on several gauge theories and discuss restrictions imposed on the structure of divergences by power counting in renormalizable cases. Together with a few well-known examples we consider the case of projectable Hořava gravity whose BRST structure is studied here for the first time. Readers interested in the general proof can skip this section and proceed directly to section 4 .

\subsection{Relativistic Yang-Mills in (3+1) dimensions}

As a first example, we consider the standard YM theory in $(3+1)$ spacetime dimensions. It has been already studied using an approach similar to ours in [35-39]. Let us start by expanding the condensed notations, ${ }^{12}$

$$
\begin{aligned}
\varphi^{a} & \mapsto A_{\mu}^{i}(x), & \varepsilon^{\alpha} & \mapsto \varepsilon^{i}(x), \\
R_{b \alpha}^{a} & \mapsto f^{i j k} \delta_{\mu}^{\nu} \delta\left(x-x_{1}\right) \delta\left(x-x_{2}\right), & P_{\alpha}^{a} & \mapsto \delta^{i j} \partial_{\mu} \delta\left(x-x_{1}\right), \\
C_{\beta \gamma}^{\alpha} & \mapsto f^{i j k} \delta\left(x-x_{1}\right) \delta\left(x-x_{2}\right), & &
\end{aligned}
$$

where $A_{\mu}^{i}(x)$ is the usual Yang-Mills field, $i$ is the color index and $f^{i j k}$ are the totally antisymmetric coordinate independent structure constants of the gauge group. We next introduce the background field $B_{\mu}^{i}(x)$ and the gauge-fixing function,

$$
\chi^{\alpha} \mapsto \partial^{\mu}\left(A_{\mu}^{i}-B_{\mu}^{i}\right)+f^{i j k} B^{j \mu}\left(A_{\mu}^{k}-B_{\mu}^{k}\right) \equiv D_{(B)}^{\mu}\left(A_{\mu}^{i}-B_{\mu}^{i}\right) .
$$

Introducing the Faddeev-Popov ghosts $\omega^{i}(x)$, antighosts $\bar{\omega}^{i}(x)$, the Lagrange multiplier $b^{i}(x)$ and the BRST sources

$$
\gamma_{a} \mapsto \gamma^{i \mu}(x), \quad \zeta_{\alpha} \mapsto \zeta^{i}(x), \quad \Omega^{a} \mapsto \Omega_{\mu}^{i}(x)
$$

we obtain the gauge-fixed action,

$$
\begin{aligned}
\Sigma_{0}= & \int \mathrm{d}^{4} x\left[\frac{1}{4 g^{2}} F_{\mu \nu}^{i} F^{i \mu \nu}+b^{i} D_{(B)}^{\mu}\left(A_{\mu}^{i}-B_{\mu}^{i}\right)-\frac{\alpha}{2} b^{i} b^{i}+D_{(B)}^{\mu} \bar{\omega}^{i} D_{(A) \mu} \omega^{i}\right. \\
& \left.+\gamma^{i \mu} D_{\mu(A)} \omega^{i}+\frac{1}{2} \zeta^{i} f^{i j k} \omega^{j} \omega^{k}+\Omega_{\mu}^{i} D_{(A)}^{\mu} \bar{\omega}^{i}+\Omega_{\mu}^{i} \gamma^{i \mu}\right] .
\end{aligned}
$$

\footnotetext{
${ }^{12}$ Where no confusion is possible, we keep a condensed notation for space-time coordinates as $x$ and their delta functions as $\delta(x)$.
} 
The constant $g$ is the gauge coupling and $\alpha$ is the gauge-fixing parameter. The field strength and covariant derivatives are defined in the standard way,

$$
\begin{aligned}
F_{\mu \nu}^{i} & =\partial_{\mu} A_{\nu}^{i}-\partial_{\nu} A_{\mu}^{i}+f^{i j k} A_{\mu}^{j} A_{\nu}^{k}, \\
D_{(A) \mu} \omega^{i} & =\partial_{\mu} \omega^{i}+f^{i j k} A_{\mu}^{j} \omega^{k},
\end{aligned}
$$

and similarly for $D_{(A) \mu} \bar{\omega}^{i}$. The $B$-covariant derivative $D_{(B) \mu} \bar{\omega}^{i}$ is given by an expression analogous to (3.2). Clearly, the action (3.4) is invariant under gauge rotations of all fields accompanied by simultaneous gauge transformations of $A_{\mu}^{i}$ and $B_{\mu}^{i}$ : these are precisely the background-gauge transformations introduced in section 2.2.

An important property of the YM theory is renormalizability. Its key prerequisite are restrictions imposed on divergences by power counting. The scaling transformations,

$$
x^{\mu} \mapsto a^{-1} x^{\mu}, \quad A_{\mu}^{i} \mapsto a A_{\mu}^{i},
$$

where $a$ is an arbitrary positive constant, leave the classical YM action invariant. We will say that $A_{\mu}^{i}$ has scaling dimension $(+1)$, whereas the dimension of $x^{\mu}$ is $(-1)$. The rest of (3.4) will be also invariant if we simultaneously scale all fields with the following dimensions,

$$
\left[A_{\mu}^{i}\right]=\left[B_{\mu}^{i}\right]=\left[\omega^{i}\right]=\left[\bar{\omega}^{i}\right]=1, \quad\left[b^{i}\right]=\left[\gamma^{i \mu}\right]=\left[\zeta^{i}\right]=\left[\Omega_{\mu}^{i}\right]=2 .
$$

The textbook analysis of divergent Feynman diagrams shows that the scaling dimensions of local counterterms needed to cancel the divergences do not exceed 4. Comparing with the BRST form (2.33) and taking into account that the generalized BRST operator $\boldsymbol{Q}$ increases the scaling dimension by 1 , we conclude that the dimensions of local operators entering into the renormalized gauge fermion $\hat{\Psi}$ do not exceed 3. Recalling further that the ghost number of $\hat{\Psi}$ is $(-1)$ we write down the most general expression compatible with these requirements,

$$
\hat{\Psi}[A, \omega, B, \hat{\gamma}, \zeta]=\int \mathrm{d}^{4} x\left(-\hat{\gamma}^{i \mu} U_{\mu}^{i}(A, B)+\zeta^{i} \omega^{j} V^{i j}\right),
$$

where $V^{i j}$ are dimensionless constants, while $U_{\mu}^{i}$ depends on $A_{\mu}^{i}$ and $B_{\mu}^{i}$ at most linearly. We observe that $\hat{\Psi}$ does not depend on $\Omega_{\mu}^{i}$ and is linear in $\gamma^{i \mu}$ and $\zeta^{i}$. As discussed in section 2.4, this implies that the field redefinition needed to bring the counterterms into the BRST form is independent of the auxiliary BRST sources, see (2.39). Positive dimensions of the YM field and ghosts further constrain this reparameterization to be linear.

\subsection{Higher-derivative relativistic gravity in $(3+1)$ dimensions}

The fields describing relativistic gravitational theories are identified as follows:

$$
\varphi^{a} \mapsto g_{\mu \nu}(x), \quad \varepsilon^{\alpha} \mapsto \varepsilon^{\mu}(x),
$$

where $g_{\mu \nu}(x)$ is the spacetime metric and $\varepsilon^{\mu}(x)$ is a vector field generating infinitesimal diffeomorphisms. The gauge transformations read,

$$
\delta_{\varepsilon} g_{\mu \nu}=\varepsilon^{\lambda} \partial_{\lambda} g_{\mu \nu}+g_{\mu \lambda} \partial_{\nu} \varepsilon^{\lambda}+g_{\nu \lambda} \partial_{\mu} \varepsilon^{\lambda}=\nabla_{(g) \mu} \varepsilon_{\nu}+\nabla_{(g) \nu} \varepsilon_{\mu},
$$


where in the last equality we have lowered the indices using the metric $g_{\mu \nu}$ and introduced the covariant derivative $\nabla_{(g)}$ constructed using this metric. The expression (3.10) implies,

$$
\begin{aligned}
R_{b \alpha}^{a} & \mapsto \delta_{\mu}^{\rho}\left[\delta_{\nu}^{\sigma}\left(\partial_{\lambda} \delta\left(x-x_{1}\right)\right) \delta\left(x-x_{2}\right)+\delta_{\lambda}^{\sigma} \delta\left(x-x_{1}\right) \partial_{\nu} \delta\left(x-x_{2}\right)\right]+\delta_{\nu}^{\rho} \delta_{\lambda}^{\sigma} \delta\left(x-x_{1}\right) \partial_{\mu} \delta\left(x-x_{2}\right), \\
P_{\alpha}^{a} & =0, \quad C_{\beta \gamma}^{\alpha} \mapsto \delta_{\lambda}^{\mu} \delta\left(x-x_{1}\right) \partial_{\nu} \delta\left(x-x_{2}\right)-\delta_{\nu}^{\mu}\left(\partial_{\lambda} \delta\left(x-x_{1}\right)\right) \delta\left(x-x_{2}\right) .
\end{aligned}
$$

We focus on the theory in $(3+1)$ dimensions including up to 4 -th order derivatives of the metric. The classical action reads

$$
S=\int \mathrm{d}^{4} x \sqrt{|g|}\left[\frac{1}{f_{1}^{2}} R_{\mu \nu} R^{\mu \nu}+\frac{1}{f_{2}^{2}} R^{2}-\frac{1}{2 \kappa^{2}} R+\frac{\Lambda}{\kappa^{2}}\right],
$$

where $|g|$ is the determinant of the metric, $R_{\mu \nu}$ is the corresponding Ricci tensor and $R \equiv R_{\mu \nu} g^{\mu \nu}$ is the Ricci scalar; $f_{1}^{2}, f_{2}^{2}, \kappa^{2}$ and $\Lambda$ are coupling constants. The quantum properties of this theory were first analyzed in [5]. The fact that the action contains fourth derivatives of the metric entails well-known problems with the physical interpretation of the theory [59]. However, this issue is irrelevant for our purposes.

Introducing the background metric $\mathfrak{g}_{\mu \nu}(x)$ we consider the gauge fixing function,

$$
\chi^{\alpha} \mapsto \chi^{\mu}=\mathfrak{g}^{\mu \lambda} \mathfrak{g}^{\nu \rho} \square_{(\mathfrak{g})} \nabla_{(\mathfrak{g}) \nu}\left(g_{\lambda \rho}-\mathfrak{g}_{\lambda \rho}\right),
$$

where $\nabla_{(\mathfrak{g})}$ and $\square_{(\mathfrak{g})}$ stand for the covariant derivatives and d'Alembertian constructed from the background metric. Introducing the fields of the BRST sector,

$$
\omega^{\alpha} \mapsto \omega^{\mu}(x), \quad \bar{\omega}_{\alpha} \mapsto \bar{\omega}_{\mu}(x), \quad b_{\alpha} \mapsto b_{\mu}(x), \quad \gamma_{a} \mapsto \gamma^{\mu \nu}(x), \quad \zeta_{\alpha} \mapsto \zeta_{\mu}(x), \quad \Omega^{a} \mapsto \Omega_{\mu \nu}(x),
$$

and the operator $O^{\alpha \beta}$,

$$
O^{\alpha \beta} \mapsto-\alpha \frac{\mathfrak{g}^{\mu \nu}}{\sqrt{|\mathfrak{g}|}} \square_{(\mathfrak{g})} \delta\left(x-x_{1}\right),
$$

we arrive at the gauge-fixed action,

$$
\begin{aligned}
\Sigma_{0}= & S\left[g_{\mu \nu}\right]+\int \mathrm{d}^{4} x\left\{b_{\mu} \chi^{\mu}+\frac{\alpha}{2} b_{\mu} \frac{\mathfrak{g}^{\mu \nu}}{\sqrt{|\mathfrak{g}|}} \square_{(\mathfrak{g})} b_{\nu}+\left(\nabla_{(\mathfrak{g}) \nu} \bar{\omega}_{\mu}\right) \mathfrak{g}^{\mu \lambda} \mathfrak{g}^{\nu \rho} \square_{(\mathfrak{g})}\left(\nabla_{(g) \lambda} \omega_{\rho}+\nabla_{(g) \rho} \omega_{\lambda}\right)\right. \\
& +\gamma^{\mu \nu}\left(\nabla_{(g) \mu} \omega_{\nu}+\nabla_{(g) \nu} \omega_{\mu}\right)+\zeta_{\mu} \omega^{\lambda} \partial_{\lambda} \omega^{\mu}+\Omega_{\mu \nu} \gamma^{\mu \nu} \\
& \left.+\Omega_{\mu \nu} \bar{\omega}_{\lambda}\left[\frac{\delta \chi^{\lambda}}{\delta \mathfrak{g}_{\mu \nu}}+\frac{\alpha}{2} \frac{\delta}{\delta \mathfrak{g}_{\mu \nu}}\left(\frac{\mathfrak{g}^{\lambda \rho}}{\sqrt{|\mathfrak{g}|}} \square_{(\mathfrak{g})}\right) b_{\rho}\right]\right\} .
\end{aligned}
$$

We have not expanded the variational derivatives in the last term as the corresponding expressions are rather lengthy and not informative. The background-gauge transformations correspond to diffeomorphisms,

$$
x^{\mu} \mapsto x^{\mu}+\varepsilon^{\mu}(x),
$$

under which $g_{\mu \nu}, \mathfrak{g}_{\mu \nu}, \omega^{\mu}, \Omega_{\mu \nu}$ transform as tensors, whereas $\bar{\omega}_{\mu}, b_{\mu}, \gamma^{\mu \nu}, \zeta_{\mu}$ transform as vector/tensor densities. For example,

$$
\begin{aligned}
& \delta_{\varepsilon} \omega^{\mu}=\varepsilon^{\lambda} \partial_{\lambda} \omega^{\mu}-\omega^{\lambda} \partial_{\lambda} \varepsilon^{\mu}, \\
& \delta_{\varepsilon} \bar{\omega}_{\mu}=\varepsilon^{\lambda} \partial_{\lambda} \bar{\omega}_{\mu}+\bar{\omega}_{\lambda} \partial_{\mu} \varepsilon^{\lambda}+\bar{\omega}_{\mu} \partial_{\lambda} \varepsilon^{\lambda},
\end{aligned}
$$


and similarly for the rest of the fields. It is straightforward to see that this is a symmetry of the action (3.16). The fact that $b_{\mu}$ is a covariant vector density explains the unusual placement of $\sqrt{|\mathfrak{g}|}$ in the denominator of the operator (3.15).

The four-derivative terms in the classical action (3.12) are invariant under rescaling

$$
x^{\mu} \mapsto a^{-1} x^{\mu},
$$

with the metric $g_{\mu \nu}$ kept intact. The same is true for the BRST-exact part of (3.16) if we assign the following scaling dimensions,

$$
\left[g_{\mu \nu}\right]=\left[\mathfrak{g}_{\mu \nu}\right]=\left[\omega^{\mu}\right]=\left[\bar{\omega}_{\mu}\right]=0, \quad\left[b_{\mu}\right]=\left[\Omega_{\mu \nu}\right]=1, \quad\left[\gamma^{\mu \nu}\right]=\left[\zeta_{\mu}\right]=3 .
$$

As in the case of YM, it can be shown ${ }^{13}$ [5] that the power-counting restricts the scaling dimensions of counterterms in the Lagrangian to be less than or equal to 4 . This again constrains the dependence of the gauge fermion on the auxiliary fields. We observe that the BRST transformations increase the scaling dimension of all fields ${ }^{14}$ by 1 . This implies that $\hat{\Psi}$ should contain local operators of dimensions not higher than 3 . Also, their ghost number must be equal to (-1). Taking into account the scaling dimensions (3.19) and the ghost charges (2.21) we obtain the most general expression,

$$
\hat{\Psi}[g, \omega, \mathfrak{g}, \hat{\gamma}, \zeta]=\int \mathrm{d}^{4} x\left(-\hat{\gamma}^{\mu \nu} U_{\mu \nu}(g, \mathfrak{g})+\zeta_{\mu} \omega^{\nu} V_{\nu}^{\mu}(g, \mathfrak{g})\right),
$$

where $U_{\mu \nu}, V_{\nu}^{\mu}$ are dimensionless functions of the quantum and background metric fields that transform covariantly under background diffeomorphisms. We observe that, similarly to YM, $\hat{\Psi}$ is linear in the BRST sources $\gamma$ and $\zeta$. However, since the scaling dimension of both metrics is zero, the coefficients in (3.20) can depend nonlinearly on $g_{\mu \nu}$ and $\mathfrak{g}_{\mu \nu}$. This implies that the field redefinition (2.39) required to restore the BRST structure of the renormalized action is genuinely nonlinear, cf. [5].

It is worth noting that the original proof of renormalizability of the theory (3.12) presented in [5] is tied to specific gauges where the structure of divergences is particularly simple due to some special features of the action. For more general gauges, ref. [5] took the cohomological structure of divergences as an assumption. Our results provide a proof of this structure for a general background gauge and, in this respect, complement the analysis of [5].

\subsection{Projectable Hořava gravity in $(d+1)$ dimensions}

Consider a $(d+1)$-dimensional spacetime with Arnowitt-Deser-Misner (ADM) decomposition of the metric,

$$
\mathrm{d} s^{2}=N^{2} \mathrm{~d} t^{2}+g_{i j}\left(\mathrm{~d} \mathbf{x}^{i}+N^{i} \mathrm{~d} t\right)\left(\mathrm{d} \mathbf{x}^{j}+N^{j} \mathrm{~d} t\right)
$$

\footnotetext{
${ }^{13}$ The choice of gauge (3.13) is important for the argument. It ensures that the propagators of the metric perturbations and ghosts fall off as the fourth power of momentum and as a consequence the degree of divergence of Feynman diagrams is consistent with the naive power counting.

${ }^{14}$ In this case it is due to the presence of derivatives acting on the transformed field, rather than the non-zero dimension of ghosts as it happens for YM.
} 
where the indices $i, j=1, \ldots, d$ denote spatial directions; ${ }^{15}$ they are raised and lowered using the spatial metric $g_{i j}$. Let us impose the so-called "projectability" constraint that the lapse $N$ is not dynamical and fix $N=1$. This constraint is compatible with a subgroup of time-dependent diffeomorphisms along spatial directions. Thus, the fields and gauge parameters are identified as follows,

$$
\varphi^{a} \mapsto g_{i j}(t, \mathbf{x}), N^{i}(t, \mathbf{x}), \quad \varepsilon^{\alpha} \mapsto \varepsilon^{i}(t, \mathbf{x}) .
$$

The gauge generators and the structure constants are given by the corresponding reduction of eqs. (3.11). The classical action is taken in the form [24, 25],

$$
S=\frac{1}{2 \kappa^{2}} \int \mathrm{d} t \mathrm{~d}^{d} \mathbf{x} \sqrt{|g|}\left(K_{i j} K^{i j}-\lambda K^{2}+\mathcal{V}\left(g_{i j}\right)\right)
$$

where

$$
K_{i j}=\frac{1}{2}\left(\dot{g}_{i j}-\nabla_{(g) i} N_{j}-\nabla_{(g) j} N_{i}\right)
$$

is the extrinsic curvature on the constant-time slices and $K \equiv K_{i j} g^{i j}$ is its trace. Here dot denotes derivative with respect to time and covariant derivatives $\nabla_{(g)}$ are constructed using the spatial metric $g_{i j} ; \kappa$ and $\lambda$ are coupling constants. The potential $\mathcal{V}$ contains all local terms invariant under spatial diffeomorphisms that can be constructed from the spatial metric $g_{i j}$ using no more than $2 d$ spatial derivatives; generically, it is a finite polynomial of the $d$-dimensional Riemann tensor and its covariant derivatives. Clearly, the action (3.23) does not possess Lorentz symmetry. On the other hand, its highest-derivative part is invariant under anisotropic (Lifshitz) scaling transformations,

$$
\mathbf{x} \mapsto a^{-1} \mathbf{x}, \quad t \mapsto a^{-d} t,
$$

with the scaling dimensions of the fields,

$$
\left[g_{i j}\right]=0, \quad\left[N^{i}\right]=d-1 .
$$

Note that different components of the gauge fields (the components of the ADM metric (3.21) in this case) have different dimensions which is a common situation in theories with Lifshitz scaling.

A background-gauge fixing procedure compatible with the scaling symmetry (3.25) was constructed in [26]. We introduce the background fields $\mathfrak{g}_{i j}(t, \mathbf{x}), \mathfrak{N}^{i}(t, \mathbf{x})$ and the combinations

$$
h_{i j}=g_{i j}-\mathfrak{g}_{i j}, \quad n^{i}=N^{i}-\mathfrak{N}^{i} .
$$

Then the gauge-fixing function reads,

$$
\chi^{\alpha} \mapsto \chi^{i}=D_{t} n^{i}+\frac{\alpha}{2} O^{i j} \mathfrak{g}^{k l}\left(\nabla_{(\mathfrak{g}) k} h_{l j}-\lambda \nabla_{(\mathfrak{g}) j} h_{k l}\right),
$$

where

$$
D_{t} n^{i}=\dot{n}^{i}-\mathfrak{N}^{k} \nabla_{(\mathfrak{g}) k} n^{i}+\nabla_{(\mathfrak{g}) k} \mathfrak{N}^{i} n^{k},
$$

\footnotetext{
${ }^{15}$ We do not use color YM indices in this subsection, so there should be no confusion with the notations of section 3.1.
} 
and the operator $O^{i j}$ has the form, ${ }^{16}$

$$
O^{i j}=(-1)^{d-1} \nabla_{(\mathfrak{g})}^{k_{1}} \ldots \nabla_{(\mathfrak{g})}^{k_{d-2}}\left(\Delta_{(\mathfrak{g})} \mathfrak{g}^{i j}+\xi \nabla_{(\mathfrak{g})}^{i} \nabla_{(\mathfrak{g})}^{j}\right) \nabla_{(\mathfrak{g}) k_{d-2}} \ldots \nabla_{(\mathfrak{g}) k_{1}}
$$

Here the covariant spatial Laplacian $\Delta_{(\mathfrak{g})}$ and all covariant derivatives $\nabla_{(\mathfrak{g})}$ are defined using the background metric $\mathfrak{g}_{i j}$ with their indices raised and lowered using the same metric; the constants $\alpha$ and $\xi$ are gauge parameters. This gauge fixing term satisfies all the requirements formulated in section 2.2: it is linear in the difference between the quantum and background fields, and covariant under simultaneous gauge transformations of these fields.

We now introduce the rest of objects entering in the BRST construction,

$$
\begin{array}{cccc}
\omega^{\alpha} \mapsto \omega^{i}(t, \mathbf{x}), & \bar{\omega}_{\alpha} \mapsto \bar{\omega}_{i}(t, \mathbf{x}), & b_{\alpha} \mapsto b_{i}(t, \mathbf{x}) & O^{\alpha \beta} \mapsto \frac{\alpha}{\sqrt{|\mathfrak{g}|}} O^{i j} \delta\left(t-t^{\prime}\right) \delta\left(\mathbf{x}-\mathbf{x}^{\prime}\right), \\
\gamma_{a} \mapsto\left\{\gamma^{i j}(t, \mathbf{x}), \gamma_{i}(t, \mathbf{x})\right\}, & \zeta_{\alpha} \mapsto \zeta_{i}(t, \mathbf{x}), & \Omega^{a} \mapsto\left\{\Omega_{i j}(t, \mathbf{x}), \Omega^{i}(t, \mathbf{x})\right\} .
\end{array}
$$

The full gauge-fixed action is lengthy and we do not write it explicitly. Importantly, with an appropriate assignment of dimensions to the fields it is invariant under the scaling transformations (3.25). By inspection of the gauge-fixing and the Faddeev-Popov ghost terms we find,

$$
\left[\omega^{i}\right]=\left[\bar{\omega}_{i}\right]=0, \quad\left[b_{i}\right]=1 .
$$

The background fields inherit the dimensions from their dynamical counterparts,

$$
\left[\mathfrak{g}_{i j}\right]=0, \quad\left[\mathfrak{N}^{i}\right]=d-1 .
$$

To determine the dimensions of the auxiliary fields $\gamma^{i j}, \gamma_{i}, \zeta_{i}$ recall that they couple to the BRST variations $s g_{i j}, s N^{i}, s \omega^{i}$ respectively. The latter have the same form as in relativistic gravity and thus contain one spatial derivative acting on the fields. This yields,

$$
\left[\boldsymbol{s} g_{i j}\right]=\left[\boldsymbol{s} \omega^{i}\right]=1, \quad\left[s N^{i}\right]=d .
$$

Then, the scale invariance of the terms $\gamma_{a} s \varphi^{a}, \zeta_{\alpha} s \omega^{\alpha}$ in the action requires

$$
\left[\gamma_{i}\right]=d, \quad\left[\gamma^{i j}\right]=\left[\zeta_{i}\right]=2 d-1
$$

Finally, the coupling $\Omega^{a} \gamma_{a}$ present in the action fixes the dimensions of $\Omega_{i j}, \Omega^{i}$,

$$
\left[\Omega_{i j}\right]=1, \quad\left[\Omega^{i}\right]=d .
$$

The results of [26] imply that in this theory the ultraviolet divergences consist of local operators with scaling dimensions not higher than $2 d$. Thus, all assumptions of section 2 are satisfied and according to section 2.4 the renormalizad action has the form (2.33). As the BRST transformations increase the dimensionality of the fields by unity, the renormalized gauge fermion $\hat{\Psi}$ appearing in (2.33) contains operators with dimensions less or equal to

\footnotetext{
${ }^{16}$ Note that $O^{i j}$ corresponds to the operator denoted by $\mathcal{O}_{i j}^{-1}$ in ref. [26].
} 
$(2 d-1)$. The general expression satisfying this property and having the ghost number $(-1)$ reads,

$$
\hat{\Psi}=\int \mathrm{d} t \mathrm{~d}^{d} \mathbf{x}\left(-\hat{\gamma}^{i j} U_{i j}(\gamma, \mathfrak{g})-\hat{\gamma}_{i} \mathrm{U}^{i}(g, N, \mathfrak{g}, \mathfrak{N})+\zeta_{i} \omega^{j} V_{j}^{i}(g, \mathfrak{g})\right),
$$

where $U_{i j}, V_{j}^{i}$ are dimensionless functions of $g_{i j}, \mathfrak{g}_{i j}$, whereas $\mathrm{U}^{i}$ can also linearly depend on $N^{i}, \mathfrak{N}^{i}$. Once more we observe that $\hat{\Psi}$ is independent of $\Omega_{i j}, \Omega^{i}$ and depends linearly on the rest of BRST sources.

Establishing the BRST structure of counterterms in the projectable Hořava gravity together with the results of ref. [26] completes the proof of renormalizability of this theory.

\subsection{General relativity as effective field theory in $(3+1)$ dimensions}

As an example of a non-renormalizable theory we consider Einstein's general relativity in $(3+1)$ dimensions. The field content and gauge transformations are the same as in section 3.2. What differs is the structure of the classical action which now reads,

$$
S=\frac{1}{2 \kappa^{2}} \int \mathrm{d}^{4} x \sqrt{|g|}(2 \Lambda-R+\ldots),
$$

where dots stand for an infinite sum of various local scalar operators constructed from the Riemann tensor and its derivatives. They can be ordered according to the total number of derivatives $n$ they contain. ${ }^{17}$ At each fixed order, the number of possible terms is finite (though it grows quickly with $n$ ). In the spirit of effective field theory, the higher derivative contributions are treated as corrections to the terms explicitly shown in (3.32). In particular, the graviton propagator is determined from the Einstein-Hilbert part and falls off as $p^{-2}$ at large momenta $p$.

The background-covariant gauge-fixing function can be chosen as in eq. (3.13). A yet simpler choice is provided by

$$
\chi^{\alpha} \mapsto \chi^{\mu}=\mathfrak{g}^{\mu \lambda} \mathfrak{g}^{\nu \rho} \nabla_{(\mathfrak{g}) \nu}\left(g_{\lambda \rho}-\mathfrak{g}_{\lambda \rho}\right),
$$

where $\mathfrak{g}_{\mu \nu}$ is the background metric. The rest of the gauge fixing construction proceeds in complete analogy with section 3.2. In the present case there are no power-counting arguments constraining the dependence of divergences on auxiliary fields. Still, the proposition formulated in section 2.4 ensures that they are compatible with the BRST structure. The field renormalization required to recover this structure is expected to have the general form (2.30) and involve ghosts and auxiliary fields in a nonlinear manner.

\section{Equations for the effective action}

We now derive the equations obeyed by the effective action $\Gamma_{L}$ defined in (2.25) corresponding to the generating functional of the form (2.32). We will omit the loop index $L$ in this section.

\footnotetext{
${ }^{17}$ Thus, the terms $R_{\mu \nu} R^{\mu \nu}$ and $R^{2}$ contain 4 derivatives $(n=4), R_{\mu \nu \lambda \rho} R^{\lambda \rho \sigma \tau} R_{\sigma \tau}{ }^{\mu \nu}$ contains 6 derivatives $(n=6)$, etc.
} 
As shown in appendix A, the closure of $\Sigma$ under the action of the extended BRST operator, $\boldsymbol{Q} \Sigma=0$, together with the absence of anomalies, implies the Slavnov-Taylor identity for the partition function,

$$
\left[-J_{a} \frac{\delta}{\delta \gamma_{a}}+\bar{\xi}_{\alpha} \frac{\delta}{\delta \zeta_{\alpha}}+\xi^{\alpha} \frac{\delta}{\delta y^{\alpha}}+\Omega^{a} \frac{\delta}{\delta \phi^{a}}\right] W=0 .
$$

Whereas the invariance of $\Sigma$ and $\Psi$ under background gauge transformations leads to the Ward identities,

$$
\begin{gathered}
{\left[-J_{a} R_{b \alpha}^{a} \frac{\delta}{\delta J_{b}}+C_{\beta \alpha}^{\gamma} \bar{\xi}_{\gamma} \frac{\delta}{\delta \bar{\xi}_{\beta}}-C_{\gamma \alpha}^{\beta} \xi^{\gamma} \frac{\delta}{\delta \xi^{\beta}}-C_{\gamma \alpha}^{\beta} y^{\gamma} \frac{\delta}{\delta y^{\beta}}\right.} \\
\left.+R_{\alpha}^{a}(\phi) \frac{\delta}{\delta \phi^{a}}-\gamma_{b} R_{a \alpha}^{b} \frac{\delta}{\delta \gamma_{a}}+C_{\gamma \alpha}^{\beta} \zeta_{\beta} \frac{\delta}{\delta \zeta_{\gamma}}+R_{b \alpha}^{a} \Omega^{b} \frac{\delta}{\delta \Omega^{a}}\right] W=0 .
\end{gathered}
$$

In addition, the equations of motion for the Lagrange multiplier $b_{\alpha}$ imply,

$$
\left[\chi_{a}^{\alpha} \frac{\delta}{\delta J_{a}}-O^{\alpha \beta} \frac{\delta}{\delta y^{\beta}}-\frac{\Omega^{a}}{2} \frac{\delta O^{\alpha \beta}}{\delta \phi^{a}} \frac{\delta}{\delta \xi^{\beta}}+y^{\alpha}\right] W=0 .
$$

Let us stress that the derivation of eqs. (4.2), (4.3) essentially relies on the property that the gauge generators and the gauge-fixing condition are linear in the quantum field.

Turning to the effective action, we use the relations (2.24), (2.26) and rewrite the identities (4.1), (4.2) and (4.3) in the following form, ${ }^{18}$

$$
\begin{array}{r}
\frac{\delta \Gamma}{\delta \gamma_{a}} \frac{\delta \Gamma}{\delta \varphi^{a}}+\frac{\delta \Gamma}{\delta \zeta_{\alpha}} \frac{\delta \Gamma}{\delta \omega^{\alpha}}+b_{\alpha} \frac{\delta \Gamma}{\delta \bar{\omega}_{\alpha}}+\Omega^{a} \frac{\delta \Gamma}{\delta \phi^{a}}=0, \\
R_{\alpha}^{a}{ }_{\alpha}(\varphi) \frac{\delta \Gamma}{\delta \varphi^{a}}-C_{\beta \alpha}^{\gamma} \omega^{\beta} \frac{\delta \Gamma}{\delta \omega^{\gamma}}+\bar{\omega}_{\beta} C_{\gamma \alpha}^{\beta} \frac{\delta \Gamma}{\delta \bar{\omega}_{\gamma}}+b_{\beta} C_{\gamma \alpha}^{\beta} \frac{\delta \Gamma}{\delta b_{\gamma}} \\
+R_{\alpha}^{a}(\phi) \frac{\delta \Gamma}{\delta \phi^{a}}-\gamma_{b} R_{a \alpha}^{b} \frac{\delta \Gamma}{\delta \gamma_{a}}+\zeta_{\beta} C_{\gamma \alpha}^{\beta} \frac{\delta \Gamma}{\delta \zeta_{\gamma}}+R_{b \alpha}^{a} \Omega^{b} \frac{\delta \Gamma}{\delta \Omega^{a}}=0, \\
\chi_{a}^{\alpha}\left(\varphi^{a}-\phi^{a}\right)-O^{\alpha \beta} b_{\beta}-\frac{\Omega^{a}}{2} \frac{\delta O^{\alpha \beta}}{\delta \phi^{a}} \bar{\omega}_{\beta}-\frac{\delta \Gamma}{\delta b_{\alpha}}=0 .
\end{array}
$$

It is convenient to consider a reduced effective action $\hat{\Gamma}$ obtained from $\Gamma$ by subtracting the gauge-fixing term and its derivatives with respect to the background fields,

$$
\hat{\Gamma}=\Gamma-b_{\alpha}\left(\chi_{a}^{\alpha}(\varphi-\phi)^{a}-\frac{1}{2} O^{\alpha \beta} b_{\beta}\right)-\Omega^{a} \bar{\omega}_{\alpha}\left(\frac{\delta \chi_{b}^{\alpha}}{\delta \phi^{a}}(\varphi-\phi)^{b}-\chi_{a}^{\alpha}-\frac{1}{2} \frac{\delta O^{\alpha \beta}}{\delta \phi^{a}} b_{\beta}\right) .
$$

Substituting this expression into (4.4c) yields that $\hat{\Gamma}$ is independent of $b_{\alpha}$,

$$
\frac{\delta \hat{\Gamma}}{\delta b_{\alpha}}=0 .
$$

Then the identity (4.4a) splits into two equations,

$$
\begin{aligned}
\chi_{a}^{\alpha} \frac{\delta \hat{\Gamma}}{\delta \gamma_{a}}+\frac{\delta \hat{\Gamma}}{\delta \bar{\omega}_{\alpha}} & =0, \\
\frac{\delta \hat{\Gamma}}{\delta \gamma_{a}} \frac{\delta \hat{\Gamma}}{\delta \varphi^{a}}+\frac{\delta \hat{\Gamma}}{\delta \zeta_{\alpha}} \frac{\delta \hat{\Gamma}}{\delta \omega^{\alpha}}+\Omega^{a}\left(\frac{\delta \hat{\Gamma}}{\delta \phi^{a}}+\bar{\omega}_{\alpha} \frac{\delta \chi_{b}^{\alpha}}{\delta \phi^{a}} \frac{\delta \hat{\Gamma}}{\delta \gamma_{b}}\right) & =0 .
\end{aligned}
$$

\footnotetext{
${ }^{18}$ Recall that we omit averaging symbols on the mean fields.
} 
The first one implies that $\hat{\Gamma}$ depends on the antighost only through the combination (2.31), so that

$$
\hat{\Gamma}=\hat{\Gamma}[\varphi, \omega, \phi, \hat{\gamma}, \zeta, \Omega]
$$

Next, we use the relation

$$
\left.\frac{\delta}{\delta \phi^{a}}\right|_{\hat{\gamma}}=\left.\frac{\delta}{\delta \phi^{a}}\right|_{\gamma}+\bar{\omega}_{\alpha} \frac{\delta \chi_{b}^{\alpha}}{\delta \phi^{a}} \frac{\delta}{\delta \gamma^{b}},
$$

where the index on the right of the vertical line means that the $\phi$-derivative is taken at fixed $\hat{\gamma}$ or $\gamma$. Consequently, eq. (4.7b) takes the form,

$$
\frac{\delta \hat{\Gamma}}{\delta \hat{\gamma}_{a}} \frac{\delta \hat{\Gamma}}{\delta \varphi^{a}}+\frac{\delta \hat{\Gamma}}{\delta \zeta_{\alpha}} \frac{\delta \hat{\Gamma}}{\delta \omega^{\alpha}}+\Omega^{a} \frac{\delta \hat{\Gamma}}{\delta \phi^{a}}=0
$$

The Ward identities (4.4b) also simplify to,

$$
R_{\alpha}^{a}(\varphi) \frac{\delta \hat{\Gamma}}{\delta \varphi^{a}}-C_{\beta \alpha}^{\gamma} \omega^{\beta} \frac{\delta \hat{\Gamma}}{\delta \omega^{\gamma}}+R_{\alpha}^{a}(\phi) \frac{\delta \hat{\Gamma}}{\delta \phi^{a}}-\hat{\gamma}_{b} R^{b}{ }_{a \alpha} \frac{\delta \hat{\Gamma}}{\delta \hat{\gamma}_{a}}+\zeta_{\beta} C_{\gamma \alpha}^{\beta} \frac{\delta \hat{\Gamma}}{\delta \zeta_{\gamma}}+R_{b \alpha}^{a} \Omega^{b} \frac{\delta \hat{\Gamma}}{\delta \Omega^{a}}=0
$$

Finally, $\hat{\Gamma}$ has zero ghost number, i.e. it is invariant under phase rotations of the fields $\omega$, $\hat{\gamma}, \zeta, \Omega$ with charges (2.21). Together with eqs. (4.10) this will be used in the next section to constrain the structure of ultraviolet divergences.

Clearly, the identities (4.10) are satisfied by the reduced tree-level action $\hat{\Sigma}_{0}$, which is related to (2.17) by a formula analogous to (4.5). Explicitly, we have,

$$
\hat{\Sigma}_{0}=S[\varphi]+\hat{\gamma}_{a} R_{\alpha}^{a}(\varphi) \omega^{\alpha}+\frac{1}{2} \zeta_{\alpha} C_{\beta \gamma}^{\alpha} \omega^{\beta} \omega^{\gamma}
$$

Note that $\hat{\Sigma}_{0}$ does not have any explicit dependence ${ }^{19}$ on $\Omega^{a}$ and $\phi^{a}$. Consequently, the last term in (4.10a) and the third term in (4.10b) are absent in the corresponding identities for $\hat{\Sigma}_{0}$.

\section{$5 \quad$ Structure of divergences}

We return to the renormalization procedure. Let us assume that at the order of $(L-1)$ loops we have already shown that the renormalized generating functional $W_{L-1}$ has the form (2.32)-(2.36). The first divergence of the effective action $\Gamma_{L-1}$ appears at order $\hbar^{L}$ and is local, see eqs. (2.27), (2.28). The standard procedure prescribes to subtract it from $\Sigma_{L-1}$ in order to obtain the action renormalized at $L$ loops. Our task is to work out the structure of this divergence. To avoid cluttered notations we will omit the indices related to the induction step and will denote the relevant divergent part $\Gamma_{L, \infty}$ simply as $\Gamma_{\infty}$.

First, we observe that the transformation (4.5) involves only finite quantities, so that the divergent parts of $\Gamma$ and $\hat{\Gamma}$ coincide,

$$
\Gamma_{\infty}=\hat{\Gamma}_{\infty}[\varphi, \omega, \phi, \hat{\gamma}, \zeta, \Omega]
$$

\footnotetext{
${ }^{19}$ Only an implicit dependence of $\hat{\Sigma}_{0}$ on $\phi^{a}$ through the combination (2.31) remains.
} 
Due to the linearity of the Ward identities (4.10b), they are obeyed separately by each term in the expansion of $\hat{\Gamma}$ in $\hbar$; in particular, they hold for the divergent part $\hat{\Gamma}_{\infty}$. Next, we consider eq. (4.10a). The first divergent contribution into it appears at the order $\hbar^{L}$. Equation (4.10a) at this order then implies

$$
\boldsymbol{Q}_{+} \hat{\Gamma}_{\infty}=0
$$

where we have introduced an operator $\boldsymbol{Q}_{+}$that acts on a functional $X$ of the fields $\varphi, \omega$, $\phi, \hat{\gamma}, \zeta, \Omega$ as follows,

$$
\begin{aligned}
\boldsymbol{Q}_{+} X & =\frac{\delta \hat{\Sigma}_{0}}{\delta \hat{\gamma}_{a}} \frac{\delta X}{\delta \varphi^{a}}+\frac{\delta \hat{\Sigma}_{0}}{\delta \varphi^{a}} \frac{\delta X}{\delta \hat{\gamma}_{a}}+\frac{\delta \hat{\Sigma}_{0}}{\delta \zeta_{\alpha}} \frac{\delta X}{\delta \omega^{\alpha}}+\frac{\delta \hat{\Sigma}_{0}}{\delta \omega^{\alpha}} \frac{\delta X}{\delta \zeta_{\alpha}}+\Omega^{a} \frac{\delta X}{\delta \phi^{a}} \\
& \equiv\left(\hat{\Sigma}_{0}, X\right)+\Omega^{a} \frac{\delta X}{\delta \phi^{a}} .
\end{aligned}
$$

Here $\hat{\Sigma}_{0}$ is the reduced tree-level action (4.11) and in the second line we defined the antibracket $\left(\hat{\Sigma}_{0}, X\right)$. A straightforward calculation using the structural relations $(2.4),(2.5)$ shows that the latter is nilpotent,

$$
\left(\hat{\Sigma}_{0},\left(\hat{\Sigma}_{0}, X\right)\right)=0
$$

and anticommutes with the operator $\Omega \delta / \delta \phi$,

$$
\left(\hat{\Sigma}_{0}, \Omega^{a} \frac{\delta X}{\delta \phi^{a}}\right)=-\Omega^{a} \frac{\delta}{\delta \phi^{a}}\left(\hat{\Sigma}_{0}, X\right) .
$$

The properties (5.4) imply nilpotency of $\boldsymbol{Q}_{+}$. Note that using the explicit form of $\Sigma_{0}$ and the BRST transformations $(2.13), \boldsymbol{Q}_{+}$can be written as

$$
\boldsymbol{Q}_{+} X=\left(\boldsymbol{s} \varphi^{a}\right) \frac{\delta X}{\delta \varphi^{a}}+\left(\boldsymbol{s} \omega^{\alpha}\right) \frac{\delta X}{\delta \omega^{\alpha}}+\left.\Omega^{a} \frac{\delta X}{\delta \phi^{a}}\right|_{\hat{\gamma}}+\frac{\delta \hat{\Sigma}_{0}}{\delta \varphi^{a}} \frac{\delta X}{\delta \hat{\gamma}_{a}}+\frac{\delta \hat{\Sigma}_{0}}{\delta \omega^{\alpha}} \frac{\delta X}{\delta \zeta_{\alpha}} .
$$

The first three terms here resemble the action of the operator $\boldsymbol{Q}$ introduced in section 2.2. However, there are a few differences. $Q$ is defined on functionals of all quantum fields $\varphi, \omega, \bar{\omega}, b$ and external backgrounds $\phi, \gamma, \zeta, \Omega$. On the other hand, $\boldsymbol{Q}_{+}$acts on functionals that are restricted to the minimal sector of quantum fields $\varphi, \omega$ and, instead of $\gamma$, depend on the combination $\hat{\gamma}$ (see (2.31)) treated as a free variable.

We now use eq. (5.2) to determine the dependence of $\hat{\Gamma}_{\infty}$ on the background fields $\phi^{a}$.

\subsection{Separating the background field dependence}

We expand $\hat{\Gamma}_{\infty}$ in powers of the auxiliary source $\Omega$,

$$
\hat{\Gamma}_{\infty}=\sum_{k} \hat{\Gamma}_{\infty,\{k\}}, \quad \hat{\Gamma}_{\infty,\{k\}}=\Omega^{a_{1}} \ldots \Omega^{a_{k}} \hat{\Gamma}_{\infty,\{k\},\left[a_{1}, \ldots, a_{k}\right]}[\varphi, \omega, \phi, \hat{\gamma}, \zeta]
$$

We assume that this sum is finite, $k \leq K$, which will be justified shortly. Substituting (5.6) into (5.2) we obtain

$$
\begin{gathered}
\Omega^{a} \frac{\delta \hat{\Gamma}_{\infty,\{K\}}}{\delta \phi^{a}}=0, \\
\Omega^{a} \frac{\delta \hat{\Gamma}_{\infty,\{k\}}}{\delta \phi^{a}}+\left(\hat{\Sigma}_{0}, \hat{\Gamma}_{\infty,\{k+1\}}\right)=0, \quad 0 \leq k \leq K-1 .
\end{gathered}
$$


As shown in appendix B, the cohomology of the operator $\Omega \delta / \delta \phi$ on the space of local functionals vanishing at $\Omega=0$ is trivial. In other words, eq. (5.7a) implies that $\hat{\Gamma}_{\infty,\{K\}}$ is represented as

$$
\hat{\Gamma}_{\infty,\{K\}}=\Omega^{a} \frac{\delta}{\delta \phi^{a}} \Upsilon_{\{K-1\}},
$$

where $\Upsilon_{\{K-1\}}$ is a local functional of ghost number $(-1)$ invariant under background-gauge transformations. Inserting this representation into (5.7b) for $k=K-1$ yields

$$
\Omega^{a} \frac{\delta}{\delta \phi^{a}}\left(\hat{\Gamma}_{\infty,\{K-1\}}-\left(\hat{\Sigma}_{0}, \Upsilon_{\{K-1\}}\right)\right)=0
$$

where we have used the property (5.4b). Again, this implies

$$
\hat{\Gamma}_{\infty,\{K-1\}}=\left(\hat{\Sigma}_{0}, \Upsilon_{\{K-1\}}\right)+\Omega^{a} \frac{\delta}{\delta \phi^{a}} \Upsilon_{\{K-2\}}
$$

By continuing this reasoning and using the properties (5.4) we obtain a representation of the type (5.10) for all $\hat{\Gamma}_{\infty,\{k\}}, 1 \leq k \leq K-1$. For $k=0$ an additional contribution appears,

$$
\hat{\Gamma}_{\infty,\{0\}}=\left(\hat{\Sigma}_{0}, \Upsilon_{\{0\}}\right)+\boldsymbol{\Gamma},
$$

where $\boldsymbol{\Gamma}[\varphi, \omega, \hat{\gamma}, \zeta]$ is independent of $\Omega$ and the background field $\phi$. Collecting all contributions together we arrive at

$$
\begin{aligned}
\hat{\Gamma}_{\infty} & =\boldsymbol{\Gamma}[\varphi, \omega, \hat{\gamma}, \zeta]+\sum_{k=0}^{K-1}\left(\hat{\Sigma}_{0}, \Upsilon_{\{k\}}\right)+\sum_{k=1}^{K} \Omega^{a} \frac{\delta}{\delta \phi^{a}} \Upsilon_{\{k-1\}} \\
& =\boldsymbol{\Gamma}[\varphi, \omega, \hat{\gamma}, \zeta]+\boldsymbol{Q}_{+} \Upsilon
\end{aligned}
$$

where in the second line we have defined

$$
\Upsilon[\varphi, \omega, \phi, \hat{\gamma}, \zeta, \Omega]=\sum_{k=0}^{K-1} \Upsilon_{\{k\}}
$$

We can now appreciate the power of the background-field approach. The pieces dependent on the background fields have separated into a $\boldsymbol{Q}_{+}$-exact contribution leaving behind the part $\boldsymbol{\Gamma}$ that depends only on the quantum fields. The original invariance under background-gauge transformations implies that $\boldsymbol{\Gamma}$ is gauge-invariant on its own. More precisely, we write

$$
\boldsymbol{\Gamma}=\boldsymbol{S}[\varphi]+\Lambda[\varphi, \omega, \hat{\gamma}, \zeta],
$$

where $\Lambda$ vanishes at $\omega=0$. The ghost-independent part $\boldsymbol{S}[\varphi]$ cannot depend on $\hat{\gamma}$ or $\zeta$ as the latter have negative ghost charges (see (2.21)), whereas the ghost number of $\boldsymbol{\Gamma}$ is zero. Then, due to the Ward identities (4.10b), the local functional $\boldsymbol{S}[\varphi]$ satisfies

$$
\frac{\delta \boldsymbol{S}}{\delta \varphi^{a}} R_{\alpha}^{a}(\varphi)=0 .
$$

We will see in section 5.3 that in the subtraction procedure it combines with the classical action $S[\varphi]$ and corresponds to the renormalization of the couplings in the classical gauge 
invariant Lagrangian. The rest of the terms in (5.12), (5.14) generates a renormalization of the gauge fermion and the corresponding field redefinition.

We still have to justify the assumption that the sum (5.6) can be truncated at finite $k$. We do it using the notion of derivative expansion. Being local, the functional $\hat{\Gamma}_{\infty}$ is a spacetime integral of a Lagrangian which can be written as a series of terms, each of them containing a finite number of derivatives. Let us introduce a formal book-keeping parameter $l_{*}$ of dimension of length counting the number of derivatives in a given term, and convert the derivative expansion into a Taylor $\operatorname{series}^{20}$ in $l_{*}$. We denote by $\hat{\Gamma}_{\infty}^{N}$ the part of $\hat{\Gamma}_{\infty}$ containing all terms of order $l_{*}^{n}, n \leq N$, i.e. all terms with up to $N$ derivatives. Now, $\Omega$ is an anticommuting local field. With a finite number of derivatives at disposal, one can construct only a finite number of local operators out of it. Therefore $\hat{\Gamma}_{\infty}^{N}$ is a finite polynomial $^{21}$ in $\Omega$.

Next, we observe that $\hat{\Sigma}_{0}$ is also a local functional and hence contains derivatives in non-negative powers. Thus, it is represented as a series with non-negative powers of $l_{*}$, so that the antibracket $\left(\hat{\Sigma}_{0}, \ldots\right)$ acting on a given operator cannot decrease its order in $l_{*}$. In addition, the operator $\Omega \delta / \delta \phi$ does not contain $l_{*}$ at all. We conclude that $\hat{\Gamma}_{\infty}^{N}$ satisfies eq. (5.2), up to corrections of order $l_{*}^{N+1}$,

$$
\boldsymbol{Q}_{+} \hat{\Gamma}_{\infty}^{N}=\mathcal{O}\left(l_{*}^{N+1}\right)
$$

Splitting $\hat{\Gamma}_{\infty}^{N}$ into monomials in $\Omega$ one can repeat the derivation leading to (5.12), up to corrections of order $\mathcal{O}\left(l_{*}^{N+1}\right)$ on the r.h.s. As this representation holds for any $N$, we can send the latter to infinity ${ }^{22}$ and recover (5.12) for the full divergent part $\hat{\Gamma}_{\infty}$ without any corrections.

\subsection{Ghost-dependent contribution}

It remains to fix the structure of the term $\Lambda$ in (5.14). It satisfies the equation,

$$
\left(\hat{\Sigma}_{0}, \Lambda\right)=0
$$

Using the explicit form of the reduced tree-level action (4.11) and the definition of the antibracket, we obtain

$$
\begin{aligned}
R_{\alpha}^{a}(\varphi) \omega^{\alpha} \frac{\delta \Lambda}{\delta \varphi^{a}}+\left(\frac{\delta S}{\delta \varphi^{a}}+\right. & \left.\hat{\gamma}_{b} R_{a \alpha}^{b} \omega^{\alpha}\right) \frac{\delta \Lambda}{\delta \hat{\gamma}_{a}}+\frac{1}{2} C_{\beta \gamma}^{\alpha} \omega^{\beta} \omega^{\gamma} \frac{\delta \Lambda}{\delta \omega^{\alpha}} \\
& +\left(-\hat{\gamma}_{a} R_{\alpha}^{a}(\varphi)+\zeta_{\beta} C_{\alpha \gamma}^{\beta} \omega^{\gamma}\right) \frac{\delta \Lambda}{\delta \zeta_{\alpha}}=0
\end{aligned}
$$

\footnotetext{
${ }^{20}$ In theories with Lifshitz scaling it would be natural to assign different weights to derivatives along different spacetime directions, cf. section 3.3. However, the argument presented below does not depend on whether one introduces such weighting or not, so for simplicity we treat all derivatives on equal footing.

${ }^{21}$ Note that its highest power is not directly related to $N$ and can depend on the specifics of the theory such as number of internal indices and spacetime dimensions, power-counting considerations, etc. The only property which is important for us here is that this power is finite.

${ }^{22}$ In renormalizable theories with finite number of coupling constants the derivative expansion usually terminates at a finite order in $N$.
} 
In addition, the invariance of $\Lambda$ with respect to background-gauge transformations implies the Ward identities (cf. (4.10b)),

$$
R_{\alpha}^{a}(\varphi) \frac{\delta \Lambda}{\delta \varphi^{a}}-C_{\beta \alpha}^{\gamma} \omega^{\beta} \frac{\delta \Lambda}{\delta \omega^{\gamma}}-\hat{\gamma}_{b} R_{a \alpha}^{b} \frac{\delta \Lambda}{\delta \hat{\gamma}_{a}}+\zeta_{\beta} C^{\beta}{ }_{\gamma \alpha} \frac{\delta \Lambda}{\delta \zeta_{\gamma}}=0
$$

Multiplying the latter expression by $\omega^{\alpha}$ and subtracting it from (5.18), we arrive at the equation

$$
\left(\boldsymbol{q}_{0}+\boldsymbol{q}_{1}\right) \Lambda=0
$$

where the operators $\boldsymbol{q}_{0,1}$ are defined as

$$
\begin{aligned}
& \boldsymbol{q}_{0} \Lambda=\frac{\delta S}{\delta \varphi^{a}} \frac{\delta \Lambda}{\delta \hat{\gamma}_{a}}-\hat{\gamma}_{a} R_{\alpha}^{a}(\varphi) \frac{\delta \Lambda}{\delta \zeta_{\alpha}} \\
& \boldsymbol{q}_{1} \Lambda=-\frac{1}{2} C_{\alpha \beta}^{\gamma} \omega^{\alpha} \omega^{\beta} \frac{\delta \Lambda}{\delta \omega^{\gamma}} .
\end{aligned}
$$

Both operators are nilpotent and anticommute with each other,

$$
\left(\boldsymbol{q}_{0}\right)^{2}=\left(\boldsymbol{q}_{1}\right)^{2}=\boldsymbol{q}_{0} \boldsymbol{q}_{1}+\boldsymbol{q}_{1} \boldsymbol{q}_{0}=0 .
$$

The operator $\boldsymbol{q}_{0}$ is known in the mathematical literature as Koszul-Tate differential [56].

Let us expand $\Lambda$ in powers of the ghost fields $\omega^{\alpha}$,

$$
\Lambda=\sum_{k=1}^{\infty} \Lambda^{\{k\}}, \quad \Lambda^{\{k\}}=\omega^{\alpha_{1}} \ldots \omega^{\alpha_{k}} \Lambda_{\left[\alpha_{1}, \ldots, \alpha_{k}\right]}^{\{k\}}[\varphi, \hat{\gamma}, \zeta]
$$

Note that the sum starts at $k=1$ as, by definition, $\Lambda$ vanishes at $\omega=0$. The conservation of the ghost number and the ghost charges (2.21) imply that each term $\Lambda^{\{k\}}$ in the expansion is a finite polynomial in $\hat{\gamma}$ and $\zeta$ that vanishes at $\hat{\gamma}=\zeta=0$. Thus $\Lambda^{\{k\}}$ satisfies,

$$
\left.\Lambda^{\{k\}}\right|_{\omega=0}=\left.\Lambda^{\{k\}}\right|_{\hat{\gamma}=\zeta=0}=0 .
$$

We now substitute (5.23) into (5.20) and obtain a chain of equations,

$$
\begin{aligned}
\boldsymbol{q}_{0} \Lambda^{\{1\}} & =0, \\
\boldsymbol{q}_{0} \Lambda^{\{k\}}+\boldsymbol{q}_{1} \Lambda^{\{k-1\}} & =0, \quad k \geq 2 .
\end{aligned}
$$

The Koszul-Tate differential $\boldsymbol{q}_{0}$ has trivial cohomology on functionals satisfying (5.24) if the gauge algebra obeys the conditions $2.1,2.1$ from section 2.1 [51]:

$$
\boldsymbol{q}_{0} X=0,\left.X\right|_{\omega=0}=\left.X\right|_{\hat{\gamma}=\zeta=0}=0 \quad \Longrightarrow \quad X=\boldsymbol{q}_{0} Y .
$$

Moreover, under natural assumptions about the regularity of the equations of motion, the functional $Y$ can be chosen to be local $[56,60]$, provided $X$ itself is local. Finally, one can show along the lines of [60] that there exists a choice of $Y$ which inherits all linearly realized symmetries commuting with $\boldsymbol{q}_{0}$. In particular, we can take $Y[\varphi, \omega, \hat{\gamma}, \zeta]$ to be invariant under background-gauge transformations if so is $X$. 
Thus we write,

$$
\Lambda^{\{1\}}=\boldsymbol{q}_{0} \Xi^{\{1\}},
$$

where $\Xi^{\{1\}}$ is local and background-gauge invariant. Substituting this into eq. (5.25b) for $k=2$ and interchanging the order of $\boldsymbol{q}_{0}$ and $\boldsymbol{q}_{1}$ we obtain,

$$
\boldsymbol{q}_{0}\left(\Lambda^{\{2\}}-\boldsymbol{q}_{1} \Xi^{\{1\}}\right)=0
$$

whence

$$
\Lambda^{\{2\}}=\boldsymbol{q}_{1} \Xi^{\{1\}}+\boldsymbol{q}_{0} \Xi^{\{2\}} .
$$

Continuing by induction, we obtain analogous representations for all $\Lambda^{\{k\}}$. Collected together they give,

$$
\Lambda=\left(\boldsymbol{q}_{0}+\boldsymbol{q}_{1}\right) \Xi, \quad \Xi=\sum_{k=1}^{\infty} \Xi^{\{k\}} .
$$

To make the last step, we notice that $\Xi$, due its invariance under background-gauge transformations, obeys a Ward identity analogous to (5.19). Combining this with (5.30) we get,

$$
\Lambda=\left(\hat{\Sigma}_{0}, \Xi\right)
$$

This is our final expression for $\Lambda$.

Putting together the contributions (5.12), (5.14), (5.31) and reintroducing the loop index $L$, we obtain the desired form of the $L$-loop divergence

$$
\Gamma_{L, \infty}=\boldsymbol{S}_{L}[\varphi]+\boldsymbol{Q}_{+} \boldsymbol{\Upsilon}_{L}
$$

where $\Upsilon_{L}=\Upsilon_{L}+\Xi_{L}$ and we have used that $\Xi_{L}$ is independent of $\phi$.

\subsection{Subtraction and field redefinition}

We now define the $L$-th order renormalized action $\operatorname{as}^{23}$ (compare with (2.29)),

$$
\Sigma_{L}[\varphi, \omega, \bar{\omega}, b, \phi, \gamma, \zeta, \Omega]=\Sigma_{L-1}-\hbar^{L} \Gamma_{L, \infty}[\varphi, \omega, \phi, \hat{\gamma}, \zeta, \Omega]+\mathcal{O}\left(\hbar^{L+1}\right),
$$

where the last term on the r.h.s. stands for local operators multiplied by at least $\hbar^{L+1}$ that will be specified shortly. The presence of these operators does not spoil the key property of the subtraction prescription, namely that it removes all subdivergences at $(L+1)$-loop order. Thus, according to the assumption stated in section 2.3, the $(L+1)$-loop divergence will be local.

We now show that $\Sigma_{L}$ can be brought to the form (2.33) by a reparameterization of the fields $\varphi, \omega$. Substituting the expression (5.32) in (5.33) and expanding explicitly the

\footnotetext{
${ }^{23}$ Strictly speaking, according to the standard scheme one should take $\phi^{a}-\delta \Psi_{L-1} / \delta \gamma_{a}$ and $\delta \Psi_{L-1} / \delta \zeta_{\alpha}$ instead of $\varphi^{a}$ and $\omega^{\alpha}$ as arguments of $\Gamma_{L, \infty}$. However, due to the representation (2.35) valid for $\Psi_{L-1}$, the difference produced by this replacement is of higher order in $\hbar$. It is included in the $\mathcal{O}\left(\hbar^{L+1}\right)$ term in (5.33).
} 
operator $\boldsymbol{Q}_{+}$we obtain,

$$
\begin{aligned}
\Sigma_{L}= & \Sigma_{0}+\sum_{l=1}^{L-1} \hbar^{l} \Sigma_{l}^{C}-\hbar^{L} \boldsymbol{S}_{L}-\hbar^{L} \frac{\delta \boldsymbol{\Upsilon}_{L}}{\delta \hat{\gamma}_{a}} \frac{\delta \hat{\Sigma}_{0}}{\delta \varphi^{a}}+\hbar^{L} \frac{\delta \boldsymbol{\Upsilon}_{L}}{\delta \zeta_{\alpha}} \frac{\delta \hat{\Sigma}_{0}}{\delta \omega^{\alpha}} \\
& -\hbar^{L} \frac{\delta \hat{\Sigma}_{0}}{\delta \hat{\gamma}_{a}} \frac{\delta \boldsymbol{\Upsilon}_{L}}{\delta \varphi^{a}}-\hbar^{L} \frac{\delta \hat{\Sigma}_{0}}{\delta \zeta_{\alpha}} \frac{\delta \boldsymbol{\Upsilon}_{L}}{\delta \omega^{\alpha}}-\left.\hbar^{L} \Omega^{a} \frac{\delta \boldsymbol{\Upsilon}_{L}}{\delta \phi^{a}}\right|_{\hat{\gamma}}+\mathcal{O}\left(\hbar^{L+1}\right)
\end{aligned}
$$

As before, the index $\hat{\gamma}$ on the partial derivative with respect to the background field in the last significant term emphasizes that it is taken at fixed $\hat{\gamma}$. The first two terms in the last line have the form,

$$
-\hbar^{L} \boldsymbol{s} \varphi^{a} \frac{\delta \boldsymbol{\Upsilon}_{L}}{\delta \varphi^{a}}-\hbar^{L} \boldsymbol{s} \omega^{\alpha} \frac{\delta \boldsymbol{\Upsilon}_{L}}{\delta \omega^{\alpha}}
$$

This suggests to define the $L$-th order gauge fermion,

$$
\Psi_{L}=\Psi_{L-1}-\hbar^{L} \boldsymbol{\Upsilon}_{L}
$$

and the $L$-th order counterterm

$$
\Sigma_{L}^{C}=-\boldsymbol{S}_{L}[\varphi]-\boldsymbol{s} \boldsymbol{\Upsilon}_{L}-\left.\Omega^{a} \frac{\delta \boldsymbol{\Upsilon}_{L}}{\delta \phi^{a}}\right|_{\gamma}=-\boldsymbol{S}_{L}[\varphi]-\boldsymbol{Q} \boldsymbol{\Upsilon}_{L}
$$

To proceed, we notice that the expressions (2.17) and (4.11) imply

$$
\frac{\delta \Sigma_{0}}{\delta \varphi^{a}}=\frac{\delta \hat{\Sigma}_{0}}{\delta \varphi^{a}}+b_{\alpha} \chi_{a}^{\alpha}+\Omega^{b} \bar{\omega}_{\alpha} \frac{\delta \chi_{a}^{\alpha}}{\delta \phi^{b}} .
$$

Further, as a consequence of the definition (2.31) we have

$$
\boldsymbol{s} \boldsymbol{\Upsilon}_{L}=\boldsymbol{s} \varphi^{a} \frac{\delta \boldsymbol{\Upsilon}_{L}}{\delta \varphi^{a}}+\boldsymbol{s} \omega^{\alpha} \frac{\delta \boldsymbol{\Upsilon}_{L}}{\delta \omega^{\alpha}}-b_{\alpha} \chi_{a}^{\alpha} \frac{\delta \boldsymbol{\Upsilon}_{L}}{\delta \hat{\gamma}_{a}}
$$

Finally, the $\phi$-derivatives at fixed $\gamma$ and $\hat{\gamma}$ are related by (4.9). Collecting all the previous expressions together, we find that eq. (5.34) simplifies to

$$
\Sigma_{L}=\Sigma_{0}+\sum_{l=1}^{L} \hbar^{l} \Sigma_{l}^{C}-\hbar^{L} \frac{\delta \boldsymbol{\Upsilon}_{L}}{\delta \gamma_{a}} \frac{\delta \Sigma_{0}}{\delta \varphi^{a}}+\hbar^{L} \frac{\delta \boldsymbol{\Upsilon}_{L}}{\delta \zeta_{\alpha}} \frac{\delta \Sigma_{0}}{\delta \omega^{\alpha}}+\mathcal{O}\left(\hbar^{L+1}\right)
$$

The first two terms already have the desired BRST form (2.33),

$$
\Sigma_{0}+\sum_{l=1}^{L} \hbar^{l} \Sigma_{l}^{C}=S[\varphi]-\sum_{l=1}^{L} \hbar^{l} \boldsymbol{S}_{L}[\varphi]+\boldsymbol{Q} \Psi_{L} .
$$

The remaining contributions are absorbed by a field redefinition, as we now demonstrate. First we perform the change of variables $\varphi, \omega \mapsto \varphi^{\prime}, \omega^{\prime}$ given by

$$
\begin{aligned}
\varphi^{a} & =\varphi^{\prime a}+\hbar^{L} \frac{\delta \boldsymbol{\Upsilon}_{L}}{\delta \gamma_{a}}\left(\varphi^{\prime}, \omega^{\prime}, \ldots\right)+\mathcal{O}\left(\hbar^{L+1}\right), \\
\omega^{\alpha} & =\omega^{\prime \alpha}-\hbar^{L} \frac{\delta \boldsymbol{\Upsilon}_{L}}{\delta \zeta_{\alpha}}\left(\varphi^{\prime}, \omega^{\prime}, \ldots\right)+\mathcal{O}\left(\hbar^{L+1}\right),
\end{aligned}
$$


where we again allow for possible local contributions of higher order in $\hbar$. Next, we Taylor expands all quantities in the differences $\left(\varphi-\varphi^{\prime}\right),\left(\omega-\omega^{\prime}\right)$. Then, the third and fourth terms in (5.38) are cancelled by the linear contribution in the series for $\Sigma_{0}$. Other terms generated by the expansion are of higher powers in $\hbar$. Notice that they are local. Thus, by properly adjusting the $\mathcal{O}\left(\hbar^{L+1}\right)$ contribution in (5.38) they can be cancelled as well.

To complete the argument we need to verify that the operators coupled to sources in the path integral have the right form (2.36) in terms of the new variables. This is done through the following chain of relations,

$$
\begin{aligned}
\tilde{\varphi}_{L-1}^{a}(\varphi, \omega, \ldots)-\phi^{a} & =-\frac{\delta \Psi_{L-1}}{\delta \gamma_{a}}(\varphi, \omega, \ldots) \\
& =\varphi^{a}-\phi^{a}+\sum_{l=1}^{L-1} \hbar^{l} \frac{\delta \boldsymbol{\Upsilon}_{l}}{\delta \gamma_{a}}(\varphi, \omega, \ldots) \\
& =\varphi^{\prime a}-\phi^{a}+\sum_{l=1}^{L-1} \hbar^{l} \frac{\delta \boldsymbol{\Upsilon}_{l}}{\delta \gamma_{a}}(\varphi, \omega, \ldots)+\hbar^{L} \frac{\delta \boldsymbol{\Upsilon}_{L}}{\delta \gamma_{a}}\left(\varphi^{\prime}, \omega^{\prime}, \ldots\right)+\mathcal{O}\left(\hbar^{L+1}\right) \\
& =\varphi^{\prime a}-\phi^{a}+\sum_{l=1}^{L} \hbar^{l} \frac{\delta \boldsymbol{\Upsilon}_{l}}{\delta \gamma_{a}}\left(\varphi^{\prime}, \omega^{\prime}, \ldots\right) \\
& =-\frac{\delta \Psi_{L}}{\delta \gamma_{a}}\left(\varphi^{\prime}, \omega^{\prime}, \ldots\right)=\tilde{\varphi}_{L}^{a}\left(\varphi^{\prime}, \omega^{\prime}, \ldots\right)-\phi^{a}
\end{aligned}
$$

where in passing to the fourth line we have assumed that the $\mathcal{O}\left(\hbar^{L+1}\right)$ terms in (5.40a) are adjusted to absorb the (local) contributions produced by the change of variables in $\Upsilon_{l}$, $1 \leq l \leq L-1$. Exactly the same reasoning applies to $\delta \Psi_{L-1} / \delta \zeta_{\alpha}$.

In the last step, we erase primes on the new variables. Thus, we have found the choice of variables in the path integral, such that eqs. (2.32)-(2.36) are satisfied at the $L$-th loop order. This statement extends to all loops by induction. This completes the proof of the proposition formulated in section 2.4 and is the main result of this work.

\section{Counterterms and nonlinear field renormalization in $O(N)$ model: ex- plicit one-loop calculation}

As an illustration of the above formalism we study one-loop counterterms in the $(1+1)$ dimensional $O(N)$-invariant sigma model. In particular, we will see the necessity of a nonlinear field renormalization to restore the BRST structure. We start with the action,

$$
S=\frac{1}{2 g^{2}} \int \mathrm{d}^{2} x \partial_{\mu} n_{i} \partial^{\mu} n^{i}
$$

where $i=1, \ldots, N ; g$ is the coupling constant and the scalar fields $n^{i}(x)$ are subject to the constraint,

$$
n^{2} \equiv \delta_{i j} n^{i} n^{j}=1
$$

The latter can be solved by expressing

$$
n^{i}=\frac{\varphi^{i}}{\sqrt{\varphi^{2}}}
$$


where the fields $\varphi^{i}(x)$ are unconstrained. The price to pay is the appearance of a gauge symmetry corresponding to the pointwise rescaling of $\varphi^{i}$,

$$
\delta_{\varepsilon} \varphi^{i}(x)=\varphi^{i}(x) \varepsilon(x)
$$

where $\varepsilon(x)$ is an arbitrary function. Clearly, the transformation (6.4) leaves $n^{i}(x)$, and hence the action, invariant. In terms of $\varphi^{i}$ the action reads,

$$
S[\varphi]=\frac{1}{2 g^{2}} \int \mathrm{d}^{2} x\left\{\frac{1}{\varphi^{2}}\left[\delta_{i j}-\frac{\varphi_{i} \varphi_{j}}{\varphi^{2}}\right] \partial_{\mu} \varphi^{i} \partial^{\mu} \varphi^{j}\right\}
$$

The gauge generator is linear in the fields,

$$
R_{b \alpha}^{a} \mapsto \delta_{j}^{i} \delta\left(x-x_{1}\right) \delta\left(x-x_{2}\right), \quad P_{\alpha}^{a}=0
$$

so this model belongs to the class of theories subject to our renormalization procedure. For the sake of convenience we set the coupling constant $g$ to one in what follows.

The local background-covariant gauge condition $\chi^{\alpha}(\varphi, \phi)$, the gauge fixing matrix $O^{\alpha \beta}(\phi)$ and its (nonlocal) inverse can be conveniently chosen in the form

$$
\begin{aligned}
\chi^{\alpha}(\varphi, \phi) & \mapsto \chi=\square\left(\frac{\phi_{i}(x)}{\phi^{2}(x)}\left(\varphi^{i}(x)-\phi^{i}(x)\right)\right)=\square\left(\frac{\phi(x) \cdot \varphi(x)}{\phi^{2}(x)}\right), \\
O^{\alpha \beta}(\phi) & \mapsto O\left(x, x^{\prime}\right)=-\square \delta\left(x-x^{\prime}\right), \quad O_{\alpha \beta}^{-1}(\phi) \mapsto O^{-1}\left(x, x^{\prime}\right)=-\frac{1}{\square} \delta\left(x-x^{\prime}\right),
\end{aligned}
$$

where we have introduced the notation for the $O(N)$-invariant scalar product,

$$
A \cdot B=\delta_{i j} A^{i} B^{i} \equiv A_{i} B^{i}
$$

The corresponding anticommuting ghost $\omega^{\alpha}$ and antighost $\bar{\omega}_{\alpha}$, as well as the Lagrange multiplier $b_{\alpha}$, are scalars with respect to the $(1+1)$-dimensional Lorentz transformations and do not carry any $O(N)$ indices, $\omega^{\alpha} \mapsto \omega(x), \bar{\omega}_{\alpha} \mapsto \bar{\omega}(x), b_{\alpha} \mapsto b(x)$. The theory is Abelian, $C_{\beta \gamma}^{\alpha}=0$, so that the BRST transform of the ghost field $\omega(x)$ vanishes and the source $\zeta_{\alpha}$ does not appear in the gauge-fixed action. Nevertheless, we have to keep the source $\zeta_{\alpha}$ in the gauge fermion to fulfil the requirement (2.35). Therefore, the tree level reduced gauge fermion equals

$$
\begin{aligned}
& \hat{\Psi}_{0}=-\hat{\gamma}_{a}\left(\varphi^{a}-\phi^{a}\right)+\zeta_{\alpha} \omega^{\alpha}=\int \mathrm{d}^{2} x\left(-\hat{\gamma}_{i}\left(\varphi^{i}-\phi^{i}\right)+\zeta \omega\right), \\
& \hat{\gamma}_{i}=\gamma_{i}-\frac{\phi_{i}}{\phi^{2}} \square \bar{\omega} .
\end{aligned}
$$

The background field independent choice (6.7b) of $O$ considerably simplifies the form of the BRST action (2.17) and moreover simplifies the result of integrating over the Lagrange multiplier $b_{\alpha}$. The effect of this integration is the replacement of the $b_{\alpha}$-dependent terms 
by the gauge breaking term quadratic in the gauge condition, after which the BRST action (2.17) takes the form (in condensed notations) ${ }^{24}$

$$
\begin{aligned}
\Sigma_{0}[\varphi, \omega, \bar{\omega}, \phi, \gamma, \Omega]= & S[\varphi]+\frac{1}{2} \chi^{\alpha}(\varphi, \phi) O_{\alpha \beta}^{-1} \chi^{\beta}(\varphi, \phi)-\bar{\omega}_{\alpha} \chi_{a}^{\alpha}(\phi) R_{\beta}^{a}(\varphi) \omega^{\beta} \\
& +\gamma_{a} R_{\alpha}^{a}(\varphi) \omega^{\alpha}+\Omega^{a} \bar{\omega}_{\alpha} \frac{\delta \chi^{\alpha}(\varphi, \phi)}{\delta \phi^{a}}+\Omega^{a} \gamma_{a} .
\end{aligned}
$$

Explicitly, the previous action reads

$$
\begin{aligned}
\Sigma_{0}[\varphi, \omega, \bar{\omega}, \phi, \gamma, \Omega]= & \int \mathrm{d}^{2} x\left\{\frac{1}{2} G_{i j} \partial_{\mu} \varphi^{i} \partial^{\mu} \varphi^{j}-\frac{1}{2} \frac{\varphi \cdot \phi}{\phi^{2}} \square\left(\frac{\varphi \cdot \phi}{\phi^{2}}\right)-\frac{\varphi \cdot \phi}{\phi^{2}}(\square \bar{\omega}) \omega\right. \\
& \left.+(\gamma \cdot \varphi) \omega+\left(\frac{\Omega \cdot \varphi}{\phi^{2}}-2 \frac{(\varphi \cdot \phi)(\Omega \cdot \phi)}{\left(\phi^{2}\right)^{2}}\right) \square \bar{\omega}+\Omega \cdot \gamma\right\} .
\end{aligned}
$$

Here $G_{i j}$ denotes the metric of the target manifold,

$$
G_{i j}=\frac{P_{i j}}{\varphi^{2}}, \quad P_{i j}=\delta_{i j}-\frac{\varphi_{i} \varphi_{j}}{\varphi^{2}},
$$

and $P_{i j}$ is a projector along the directions orthogonal to $\varphi^{j}$. All terms in the Lagrangian have mass dimension 2 if the dimensions of the fields are chosen as,

$$
[\varphi]=[\phi]=[\omega]=[\bar{\omega}]=[\Omega]=0, \quad[\gamma]=2 .
$$

The theory is renormalizable, hence all divergences also have dimension 2. This implies that the renormalized gauge fermion $\hat{\Psi}$ should remain linear in $\hat{\gamma}_{i}$ and independent ${ }^{25}$ of $\Omega^{i}$, as in other renormalizable examples encountered in section 3. On the other hand, due to the zero mass dimension of the gauge fields, we expect that it will have nonlinear dependence on $\varphi^{i}$ and $\phi^{i}$. These expectations are confirmed below by an explicit calculation.

The one-loop effective action of the model is given by the functional supertrace,

$$
\Gamma_{1}=\frac{1}{2} \operatorname{STr}\left(\log F_{I J}\right)
$$

where $F_{I J}$ is the inverse propagator of the theory. The latter is given by the second order mixed (left and right) functional derivatives of the action with respect to the full set of boson-fermion fields of the theory $\Phi^{I}(x)=\left(\varphi^{i}(x), \omega(x), \bar{\omega}(x)\right)$

$$
F_{I J} \delta\left(x-x^{\prime}\right)=\frac{\vec{\delta}}{\delta \Phi^{I}(x)} \Sigma_{0}[\varphi, \omega, \bar{\omega}, \phi, \gamma, \Omega] \frac{\overleftarrow{\delta}}{\delta \Phi^{J}\left(x^{\prime}\right)}
$$

This second order differential operator acting in the space of perturbations of the fields $\delta \Phi^{J}$ has the form,

$$
F_{I J}=D_{I J} \square+2 \Gamma_{I J}^{\mu} \partial_{\mu}+\Pi_{I J}
$$

\footnotetext{
${ }^{24}$ We disregard the one-loop functional determinant $(\operatorname{Det} O)^{-1 / 2}$ originating from this integration, because it is a trivial field-independent normalization constant.

${ }^{25}$ Recall that $\hat{\Psi}$ has ghost number $(-1)$, whereas the fields have ghost charges $(2.21)$. Note also that the contribution involving $\zeta$ in $\hat{\Psi}$ does not get renormalized since $\zeta$ does not appear in the action.
} 
The expressions for the matrix valued coefficients $D_{I J}, \Gamma_{I J}^{\mu}$ and $\Pi_{I J}$ are given in appendix C.

The divergent part of (6.14) for a general operator of the form (6.16) is easily obtained by the heat kernel method as a local functional of the operator coefficients [27, 28, 30, 44]. First, the inverse propagator is converted into the form of a covariant d'Alembertian,

$$
\boldsymbol{F}_{J}^{I}=-\left(\mathcal{D}_{\mu} \mathcal{D}^{\mu}\right)_{J}^{I}+\boldsymbol{P}_{J}^{I}
$$

built in terms of covariant derivatives $\mathcal{D}_{\mu}$ with some generic connection $\boldsymbol{\Gamma}_{\mu}=\boldsymbol{\Gamma}_{\mu J}^{I}$. These covariant derivatives act in the linear space of fields $\Phi=\Phi^{I}(x)$ and field matrices $\boldsymbol{X}=$ $\boldsymbol{X}_{J}^{I}(x)$ as

$$
\mathcal{D}_{\mu} \Phi=\partial_{\mu} \Phi+\boldsymbol{\Gamma}_{\mu} \Phi, \quad \mathcal{D}_{\mu} \boldsymbol{X}=\partial_{\mu} \boldsymbol{X}+\left[\boldsymbol{\Gamma}_{\mu}, \boldsymbol{X}\right] .
$$

In the case of a $(1+1)$-dimensional flat spacetime the one-loop divergence takes a particularly simple form: it depends only on the potential term $\boldsymbol{P}$ of this operator

$$
\left.\frac{1}{2} \mathrm{STr} \log \boldsymbol{F}\right|_{\infty}=\frac{1}{4 \pi(2-d)} \int \mathrm{d}^{2} x \operatorname{str} \boldsymbol{P}
$$

Here str is the matrix supertrace over indices $I$,

$$
\operatorname{str} \boldsymbol{P}=\sum_{I}(-1)^{\epsilon_{I}} \boldsymbol{P}_{I}^{I}
$$

where $\epsilon_{I}=0,1$ is the Grassmann parity of the matrix entry labeled by the index $I$. We used dimensional regularization to capture the divergence in the limit $d \rightarrow 2$.

We convert (6.16) into the form (6.17) by canonically normalizing the second-order derivative term of the inverse propagator, $F_{I J}=-D_{I K} \boldsymbol{F}_{J}^{K}$. Then

$$
\boldsymbol{F}=-\left(\square \mathbf{1}+2 \boldsymbol{\Gamma}^{\mu} \partial_{\mu}+\boldsymbol{\Pi}\right), \quad\left(\boldsymbol{\Gamma}^{\mu}\right)_{J}^{I}=D^{I K} \Gamma_{K J}^{\mu}, \quad \boldsymbol{\Pi}_{J}^{I}=D^{I K} \Pi_{K J},
$$

where $D^{I K}$ is the inverse of the matrix $D_{I J}, D^{I K} D_{K J}=\delta_{J}^{I}$. Next, the first-order derivative term of (6.21) is absorbed into the covariant derivative (6.18) with the connection $\boldsymbol{\Gamma}_{\mu}$. As a result, the operator (6.21) takes the form (6.17) with $\boldsymbol{P}=-\boldsymbol{\Pi}+\partial_{\mu} \boldsymbol{\Gamma}^{\mu}+\boldsymbol{\Gamma}^{\mu} \boldsymbol{\Gamma}_{\mu}$, so that finally the one-loop divergence reads

$$
\Gamma_{1, \infty}=-\frac{1}{4 \pi(2-d)} \int \mathrm{d}^{2} x \operatorname{str}\left(\boldsymbol{\Pi}-\boldsymbol{\Gamma}^{\mu} \boldsymbol{\Gamma}_{\mu}\right),
$$

where we have dropped the total derivative term ${ }^{26} \partial_{\mu}\left(\operatorname{str} \boldsymbol{\Gamma}^{\mu}\right)$. The matrices $\boldsymbol{\Gamma}^{\mu}$ and $\boldsymbol{\Pi}$ are

\footnotetext{
${ }^{26}$ We also disregard the ultralocal contribution of the transition from $F_{I J}$ to $\boldsymbol{F}$,

$\mathrm{S} \operatorname{Tr} \log F_{I J}=\mathrm{S} \operatorname{Tr} \log \boldsymbol{F}+\mathrm{S} \operatorname{Tr} \log \left(-D_{I J}\right)=\mathrm{S} \operatorname{Tr} \log \boldsymbol{F}+\delta(0)(\ldots)$,

which might be canceled by an appropriate local contribution of the measure in the path integral and anyway vanishes in dimensional regularization.
} 
evaluated in appendix C. Substituting the corresponding expressions into (6.22) we obtain,

$$
\begin{aligned}
\hbar \Gamma_{1, \infty}= & -\frac{\hbar}{2 \pi(2-d)} \int \mathrm{d}^{2} x\left\{\frac{N-2}{2} G_{i j} \partial_{\mu} \varphi^{i} \partial^{\mu} \varphi^{j}+\frac{\left(\phi^{2}\right)^{2}}{(\varphi \cdot \phi)^{2}}(\varphi \cdot \hat{\gamma}) \omega\right. \\
& +\left(\frac{\delta_{i j}}{\varphi^{2}}-2 \frac{\phi_{i} \varphi_{j}}{(\varphi \cdot \phi) \varphi^{2}}+\frac{\phi_{i} \phi_{j}}{(\varphi \cdot \phi)^{2}}\right) \partial_{\mu} \varphi^{i} \partial^{\mu} \varphi^{j}-\left(\frac{\delta_{i j}}{(\varphi \cdot \phi)}-\frac{\phi_{i} \varphi_{j}}{(\varphi \cdot \phi)^{2}}\right) \partial_{\mu} \varphi^{i} \partial^{\mu} \phi^{j} \\
& \left.-\left[\frac{\varphi^{2}}{(\varphi \cdot \phi)} \delta_{k}^{i}-2 \frac{\phi^{2}}{(\varphi \cdot \phi)^{2}} \varphi^{i} \phi_{k}-\frac{\varphi^{2}}{(\varphi \cdot \phi)^{2}}\left(\phi^{i} \varphi_{k}+\varphi^{i} \phi_{k}\right)+\frac{\phi^{2}\left(\varphi^{2}+\phi^{2}\right)}{(\varphi \cdot \phi)^{3}} \varphi^{i} \varphi_{k}\right] \Omega^{k} \hat{\gamma}_{i}\right\} .
\end{aligned}
$$

If we set $\phi^{i}=\varphi^{i}, \Omega^{i}=\gamma_{i}=\omega=0$, only the first term in this expression will survive corresponding to the well-known expression for the 1-loop divergence in the $O(N)$-model (see e.g. [61]).

Let us look at the terms in the last line of (6.23) bilinear in $\Omega^{k}$ and $\hat{\gamma}_{i}$. According to eq. (5.32), they originate from the action of the operator $\Omega \delta / \delta \phi$ on the one-loop $(L=1)$ quantum dressing $\boldsymbol{\Upsilon}_{1}$ of the gauge fermion in (5.36a). Clearly, we are in the situation when this dressing is independent of $\Omega$ and linear in $\hat{\gamma}$,

$$
\boldsymbol{\Upsilon}_{1}=\hat{\gamma}_{a} \boldsymbol{u}_{1}^{a}(\varphi, \phi)
$$

Therefore, the terms bilinear in $\Omega^{k}$ and $\hat{\gamma}_{i}$ should be identified with $\Omega^{a} \hat{\gamma}_{b} \delta \boldsymbol{u}_{1}^{b} / \delta \phi^{a}$, or

$$
\begin{aligned}
\frac{\partial \boldsymbol{u}_{1}^{i}(\varphi, \phi)}{\partial \phi^{k}}= & \frac{1}{2 \pi(2-d)}\left[\frac{\varphi^{2}}{\varphi \cdot \phi} \delta_{k}^{i}-\frac{2 \phi^{2}}{(\varphi \cdot \phi)^{2}} \varphi^{i} \phi_{k}\right. \\
& \left.-\frac{\varphi^{2}}{(\varphi \cdot \phi)^{2}}\left(\phi^{i} \varphi_{k}+\varphi^{i} \phi_{k}\right)+\frac{\phi^{2}\left(\varphi^{2}+\phi^{2}\right)}{(\varphi \cdot \phi)^{3}} \varphi^{i} \varphi_{k}\right]
\end{aligned}
$$

One can check that a nontrivial integrability condition for this equation is satisfied, and the solution reads

$$
\boldsymbol{u}_{1}^{i}(\varphi, \phi)=-\frac{1}{4 \pi(2-d)}\left[\frac{\phi^{2}\left(\varphi^{2}+\phi^{2}\right)}{(\varphi \cdot \phi)^{2}} \varphi^{i}-\frac{2 \varphi^{2}}{(\varphi \cdot \phi)} \phi^{i}\right] .
$$

According to (2.37), (2.38a) this function generates the one-loop field renormalization,

$$
\varphi^{i} \mapsto \tilde{\varphi}_{1}^{i}=\varphi^{i}+\hbar \boldsymbol{u}_{1}^{i}(\varphi, \phi)
$$

Notice that this renormalization is essentially nonlinear. Still, it is covariant with respect to simultaneous gauge transformations of both quantum and background fields, as it should be.

It remains to be shown that the rest of the terms in (6.23) recover the correct BRST structure of the renormalized action after the field redefinition (6.26). We observe that the first term of (6.23) is the gauge invariant counterterm — proportional to the classical action,

$$
\boldsymbol{S}_{1}=-\frac{N-2}{4 \pi(2-d)} \int \mathrm{d}^{2} x G_{i j} \partial_{\mu} \varphi^{i} \partial^{\mu} \varphi^{j}
$$


The second term bilinear in $\hat{\gamma}_{i}$ and $\omega$ can be represented as the sum of two terms:

$$
\begin{gathered}
\hat{\gamma}_{a} \frac{\delta R_{\alpha}^{a}}{\delta \varphi^{b}} \boldsymbol{u}_{1}^{b} \omega^{\alpha}=\int \mathrm{d}^{2} x\left(\hat{\gamma} \cdot \boldsymbol{u}_{1}\right) \omega=\frac{1}{4 \pi(2-d)} \int \mathrm{d}^{2} x\left[-\frac{\phi^{2}\left(\varphi^{2}+\phi^{2}\right)}{(\varphi \cdot \phi)^{2}}(\hat{\gamma} \cdot \varphi)+\frac{2 \varphi^{2}}{(\varphi \cdot \phi)}(\hat{\gamma} \cdot \phi)\right] \omega, \\
-\hat{\gamma}_{a} \frac{\delta \boldsymbol{u}_{1}^{a}}{\delta \varphi^{b}} R_{\alpha}^{b} \omega^{\alpha}=-\int \mathrm{d}^{2} x \hat{\gamma}_{i} \frac{\partial \boldsymbol{u}_{1}^{i}}{\partial \varphi^{k}} \varphi^{k} \omega=\frac{1}{4 \pi(2-d)} \int \mathrm{d}^{2} x\left[\frac{\phi^{2}\left(\varphi^{2}-\phi^{2}\right)}{(\varphi \cdot \phi)^{2}}(\hat{\gamma} \cdot \varphi)-\frac{2 \varphi^{2}}{(\varphi \cdot \phi)}(\hat{\gamma} \cdot \phi)\right] \omega .
\end{gathered}
$$

Finally, the second line of (6.23) coincides with the change of the classical action under the field reparametrization (6.26),

$$
\begin{aligned}
\frac{\delta S}{\delta \varphi^{a}} \boldsymbol{u}_{1}^{a}= & -\frac{1}{2 \pi(2-d)} \int \mathrm{d}^{2} x\left[\left(\frac{\delta_{i j}}{\varphi^{2}}-2 \frac{\phi_{i} \varphi_{j}}{(\varphi \cdot \phi) \varphi^{2}}+\frac{\phi_{i} \phi_{j}}{(\varphi \cdot \phi)^{2}}\right) \partial_{\mu} \varphi^{i} \partial^{\mu} \varphi^{j}\right. \\
& \left.-\left(\frac{\delta_{i j}}{(\varphi \cdot \phi)}-\frac{\phi_{i} \varphi_{j}}{(\varphi \cdot \phi)^{2}}\right) \partial_{\mu} \varphi^{i} \partial^{\mu} \phi^{j}\right] .
\end{aligned}
$$

With the field reparametrization (6.26) we therefore have

$$
\begin{aligned}
\left.\Sigma_{0}\right|_{\varphi \rightarrow \varphi+\hbar \boldsymbol{u}_{1}}= & S+\boldsymbol{Q} \Psi_{0}+\hbar\left(\frac{\delta S}{\delta \varphi^{a}} \boldsymbol{u}_{1}^{a}+b_{\alpha} \chi_{a}^{\alpha} \boldsymbol{u}_{1}^{a}+\hat{\gamma}_{a} \frac{\delta R_{\alpha}^{a}}{\delta \varphi^{b}} \boldsymbol{u}_{1}^{b} \omega^{\alpha}+\Omega^{a} \frac{\delta \chi_{b}^{\alpha}}{\delta \phi^{a}} \boldsymbol{u}_{1}^{b} \bar{\omega}_{\alpha}\right) \\
& +\mathcal{O}\left(\hbar^{2}\right), \\
\left.\hbar \Gamma_{1, \infty}\right|_{\varphi \rightarrow \varphi+\hbar \boldsymbol{u}_{1}}= & \hbar\left(\boldsymbol{S}_{1}+\frac{\delta S}{\delta \varphi^{a}} \boldsymbol{u}_{1}^{a}+\Omega^{a} \hat{\gamma}_{b} \frac{\delta \boldsymbol{u}_{1}^{b}}{\delta \phi^{a}}+\hat{\gamma}_{a} \frac{\delta R_{\alpha}^{a}}{\delta \varphi^{b}} \boldsymbol{u}_{1}^{b} \omega^{\alpha}-\hat{\gamma}_{a} \frac{\delta \boldsymbol{u}_{1}^{a}}{\delta \varphi^{b}} R_{\alpha}^{b} \omega^{\alpha}\right)+\mathcal{O}\left(\hbar^{2}\right) .
\end{aligned}
$$

Thus, the renormalized action reads

$$
\Sigma_{1} \equiv\left[\Sigma_{0}-\hbar \Gamma_{1, \infty}\right]_{\varphi \rightarrow \varphi+\hbar \boldsymbol{u}_{1}}=S[\varphi]-\hbar \boldsymbol{S}_{1}[\varphi]+\boldsymbol{Q}\left(\Psi_{0}-\hbar \boldsymbol{\Upsilon}_{1}\right)+\mathcal{O}\left(\hbar^{2}\right)
$$

where in the expression for $\boldsymbol{Q} \boldsymbol{\Upsilon}_{1}$ we took into account the dependence of $\hat{\gamma}_{a}=\gamma_{a}-\bar{\omega}_{\alpha} \chi_{a}^{\alpha}(\phi)$ on $\bar{\omega}$ and $\phi$. This BRST structure of the one-loop renormalization is in full agreement with (2.33) - the renormalized gauge invariant action $S_{1}[\varphi]=S[\varphi]-\hbar \boldsymbol{S}_{1}[\varphi]$ plus the BRST exact term with the gauge fermion dressed by a local quantum correction inducing the field reparameterization.

\section{Conclusions and discussion}

In this paper we have demonstrated the local BRST structure of renormalization in a wide class of gauge field theories admitting background-covariant gauges. Simply stated, we have shown that, for theories of this class, the renormalization procedure does not spoil gauge invariance. This class encompasses all standard Einstein-YM-Maxwell theories, whether renormalizable or not. In this way we reproduce the classical results concerning renormalization of Einstein-YM theories and strengthen them for the case of theories with Abelian subgroups. Other representatives of the class covered by our analysis are non-relativistic YM-Maxwell theories and projectable Hořava gravity. This offers the first demonstration 
of the BRST structure of projectable Hořava gravity which completes the proof of its renormalizability. The previous list of applications of our results is certainly not exhaustive. As suggested by the example considered in section 6 , they can be useful for studying various $\sigma$-models and other theories where gauge invariance is introduced as a tool to resolve the complicated structure of the field configuration space.

Our argument makes essential use of the background fields $\phi$. With a suitable choice of the gauge condition they allowed us to introduce an additional gauge invariance with respect to background gauge transformations. We then extended the BRST construction with an auxiliary anticommuting source $\Omega$ controlling the dependence of the gauge-fixing term on the background fields. The counterterms generated by renormalization were shown to belong to the local cohomology of the extended BRST operators on the space of functionals polynomial in $\Omega$ and the Faddeev-Popov ghosts. Our key observation is that the presence of linearly realized background-gauge invariance allows one to split the computation of this cohomology into several steps involving cohomologies of a few simpler operators. By completing these steps we have concluded that the counterterms split into a BRST exact piece and a local gauge invariant functional $\boldsymbol{S}[\varphi]$ depending only on the dynamical "quantum" - fields which renormalizes the physical action of the system. Our derivation is self-contained and does not rely on any power counting considerations. We have discussed the simplifications that appear if such considerations apply. Our results agree with those of [45] whenever they overlap.

We have discussed in detail the local field redefinition bringing the renormalized action into the BRST form. This field redefinition, which in simple models has a multiplicative linear nature, becomes essentially nonlinear in generic theories, as we illustrated with an explicit example (section 6). Despite this complication, it preserves a universal structure: at any order in the loop expansion, the renormalized quantum fields are generated by eq. (2.36) with the local generating functional $\Psi$. The latter is identical to the gauge fermion appearing in the exact part $\boldsymbol{Q} \Psi$ of the full BRST action $\Sigma=S[\varphi]+\boldsymbol{Q} \Psi$ dressed by loop corrections. This property provides a systematic algorithm to construct the field redefinition order by order in perturbation theory. What one needs to do is just to determine $\Psi$ from the part of the counterterm containing the BRST sources and background fields. This procedure becomes particularly efficient when there are additional constraints, e.g. due to power counting, that prevent $\Psi$ from depending on the BRST source $\Omega$ associated to background fields. In that case, our results imply that the $\Omega$-dependent part of the counterterm has the form $\Omega \delta \Psi / \delta \phi$ (see the definition of $\boldsymbol{Q}$ in (2.16)). Therefore, $\Psi$ can be found by simply integrating the coefficient in front of $\Omega$ with respect to the background fields. In terms of renormalized fields, the physical part $S[\varphi]$ of the renormalized action becomes gauge invariant. Thus, the divergences contained in $S[\varphi]$ have the same structure as the terms in the tree-level action and are absorbed by renormalization of the physical coupling constants.

It is worth reviewing the various assumptions about the gauge algebra that entered into our derivation. An essential assumption is the linearity of the gauge generators in the gauge fields which allows one to easily construct background-covariant gauge conditions. Moreover, the linearity of the resulting background-gauge covariance is crucial for 
its preservation at the quantum level. Another essential requirement is local completeness of the gauge generators expressed by eqs. (2.6), (2.7). This plays an important role in the homological analysis of the Koszul-Tate differential performed in $[56,60]$ and whose results we used in section 5.2. On the other hand, it appears likely that the irreducibility condition 2.1 from section 2.1 can be relaxed at the price of considerably complicating the ghost sector. Indeed, the main steps in the proof in section 5 would be unchanged, including the results of $[56,60]$ that are straightforwardly generalized to the reducible case. Finally, we assumed the gauge algebra to close off-shell which allowed us to use the standard BRST construction for the gauge fixing. It would be interesting to extend our analysis to gauge theories with open algebras. The close connection between our approach and the Batalin-Vilkovisky generalization of the BRST formalism to open algebras [48-51] makes the existence of such extension quite plausible.

Though we have not addressed this topic in the present paper, we believe that our method can be efficiently applied to renormalization of composite operators in gauge theories. Another aspect of renormalization that has been left outside the scope of this paper is that of quantum gauge anomalies. These are known to be related to BRST cohomologies with non-vanishing ghost number. It would be interesting to see if the background-field approach along the lines developed here can shed new light on this topic. We leave this study for future.

\section{Acknowledgments}

We are indebted to Frank Ferrari, Elias Kiritsis and Igor Tyutin for stimulating discussions. We thank Ioseph Buchbinder and Marc Henneaux for valuable comments on the first version of the paper. A.B. and C.S. are grateful for hospitality of the CERN Theoretical Physics Department where part of this work has been completed. This work was supported by the RFBR grant No.17-02-00651 (A.B. and S.S.), the Tomalla Foundation (M.H.-V.) and the Swiss National Science Foundation (S.S.).

\section{A Derivation of Slavnov-Taylor and Ward identities}

To obtain the Slavnov-Taylor identity (4.1), note that the total action including the source term in the exponential of (2.32) can be written in a BRST invariant form. For this purpose we introduce the "doubly extended" BRST operator

$$
\boldsymbol{Q}_{\mathrm{ext}}=\boldsymbol{s}+\Omega \frac{\delta}{\delta \phi}-J \frac{\delta}{\delta \gamma}+\bar{\xi} \frac{\delta}{\delta \zeta}+\xi \frac{\delta}{\delta y}, \quad \boldsymbol{Q}_{\mathrm{ext}}^{2}=0,
$$

and notice that the source term in the non-minimal sector can also be rewritten as a BRST-exact expression,

$$
\xi \bar{\omega}+y b=\left(s+\xi \frac{\delta}{\delta y}\right) y \bar{\omega}=Q_{\mathrm{ext}}(y \bar{\omega})
$$

where for brevity we omit the condensed indices of all quantities. Therefore, the total BRST action including all sources takes a compact form in terms of the extended gauge 
fermion $\Psi_{\text {ext }}$,

$$
\Sigma_{\text {ext }}=\Sigma-J \frac{\delta \Psi}{\delta \gamma}+\bar{\xi} \frac{\delta \Psi}{\delta \zeta}+\xi \bar{\omega}+y b=S+\boldsymbol{Q}_{\mathrm{ext}} \Psi_{\mathrm{ext}}, \quad \Psi_{\mathrm{ext}} \equiv \Psi+y \bar{\omega},
$$

and the path integral for the generating functional (2.32) reads

$$
e^{-W / \hbar}=\int d \Phi e^{-\Sigma_{\text {ext }} / \hbar}
$$

Clearly,

$$
\boldsymbol{Q}_{\mathrm{ext}} e^{-\Sigma_{\mathrm{ext}} / \hbar}=0
$$

or

$$
\left(-J \frac{\delta}{\delta \gamma}+\bar{\xi} \frac{\delta}{\delta \zeta}+\xi \frac{\delta}{\delta y}+\Omega \frac{\delta}{\delta \phi}\right) e^{-\Sigma_{\mathrm{ext}} / \hbar}=-\boldsymbol{s} e^{-\Sigma_{\mathrm{ext}} / \hbar}
$$

whence

$$
\left(-J \frac{\delta}{\delta \gamma}+\bar{\xi} \frac{\delta}{\delta \zeta}+\xi \frac{\delta}{\delta y}+\Omega \frac{\delta}{\delta \phi}\right) e^{-W / \hbar}=-\int d \Phi s e^{-\sum_{\mathrm{ext}} / \hbar}
$$

The path integral here has the form,

$$
\int d \Phi\left(s \Phi^{I}\right) \frac{\delta}{\delta \Phi^{I}} e^{-F[\Phi]}=-\int d \Phi\left(\frac{\delta}{\delta \Phi^{I}} s \Phi^{I}(\Phi)\right) e^{-F[\Phi]}
$$

where the expression in brackets on the r.h.s. is the variation of the integration measure $d \Phi$ under the BRST variation of the fields. It vanishes according to the assumption of anomaly-free regularization and we arrive at eq. (4.1).

For the derivation of the Ward identity (4.2), we introduce, together with the quantum fields $\Phi$ and background fields $\phi$, also the collective notations for all the sources

$$
\mathcal{J}=J_{a}, \bar{\xi}_{\alpha}, \xi^{\alpha}, y^{\alpha}, \gamma_{a}, \zeta_{\alpha}, \Omega^{a}
$$

Then, in view of our choice of background-covariant gauge conditions, $\Sigma_{\text {ext }}[\Phi, \phi, \mathcal{J}]$ is invariant with respect to the background-gauge transformations ${ }^{27}(2.10),(2.20)$ supplemented by

$$
\delta_{\varepsilon} J_{a}=-J_{b} R_{a \alpha}^{b} \varepsilon^{\alpha}, \quad \delta_{\varepsilon} \bar{\xi}_{\alpha}=\bar{\xi}_{\beta} C_{\alpha \gamma}^{\beta} \varepsilon^{\gamma}, \quad \delta_{\varepsilon} \xi^{\alpha}=-C_{\beta \gamma}^{\alpha} \xi^{\beta} \varepsilon^{\gamma}, \quad \delta_{\varepsilon} y^{\alpha}=-C_{\beta \gamma}^{\alpha} y^{\beta} \varepsilon^{\gamma} .
$$

We have,

$$
\delta_{\varepsilon} \Sigma_{\text {ext }}=\left(\delta_{\varepsilon} \Phi \frac{\delta}{\delta \Phi}+\delta_{\varepsilon} \phi \frac{\delta}{\delta \phi}+\delta_{\varepsilon} \mathcal{J} \frac{\delta}{\delta \mathcal{J}}\right) \Sigma_{\text {ext }}=0
$$

\footnotetext{
${ }^{27}$ Note that the invariance of the source term $-J_{a} \delta \Psi / \delta \gamma_{a}=J_{a}\left(\tilde{\varphi}_{L}-\phi\right)^{a}$ relies on the homogeneity of the linear transformation law for $\left(\tilde{\varphi}_{L}-\phi\right)^{a}$ contragredient to the transformation of $J_{a}$ in $(2.20)$.
} 
Next, we perform the change of integration variables $\Phi \rightarrow \Phi+\delta_{\varepsilon} \Phi$ in the path integral (A.4). If, as we did before, we disregard the gauge variation of the integration measure, we obtain the following integral identity,

$$
\int d \Phi \delta_{\varepsilon} \Phi \frac{\delta \Sigma_{\text {ext }}}{\delta \Phi} e^{-\Sigma_{\mathrm{ext}} / \hbar}=0
$$

On account of eq. (A.11), its 1.h.s. equals

$$
\int d \Phi\left(\delta_{\varepsilon} \phi \frac{\delta}{\delta \phi}+\delta_{\varepsilon} \mathcal{J} \frac{\delta}{\delta \mathcal{J}}\right) e^{-\Sigma_{\mathrm{ext}} / \hbar}=\left(\delta_{\varepsilon} \phi \frac{\delta}{\delta \phi}+\delta_{\varepsilon} \mathcal{J} \frac{\delta}{\delta \mathcal{J}}\right) e^{-W / \hbar}
$$

because the operator $\delta_{\varepsilon} \phi \delta / \delta \phi+\delta_{\varepsilon} \mathcal{J} \delta / \delta \mathcal{J}$ is independent of the integration fields $\Phi$ and can be commuted with the integration sign. Therefore,

$$
\left(\delta_{\varepsilon} \phi \frac{\delta}{\delta \phi}+\delta_{\varepsilon} \mathcal{J} \frac{\delta}{\delta \mathcal{J}}\right) W=0
$$

which in view of the expressions (2.10), (2.20) and (A.10) for $\delta_{\varepsilon} \phi$ and $\delta_{\varepsilon} \mathcal{J}$ is just the expression (4.2).

\section{B Homology of the operator $\Omega \delta / \delta \phi$}

In this appendix we prove the statement used in section 5.1 that the cohomology of the operator $\Omega \delta / \delta \phi$ on the space of local functionals vanishing at $\Omega=0$ is trivial.

Lemma. Let $X[\varphi, \phi, \Omega, \ldots]$ be a local functional of the gauge fields $\varphi^{a}$, background fields $\phi^{a}$, anticommuting BRST sources $\Omega^{a}$ and, possibly, other fields represented by dots. Assume that $X$ vanishes at $\Omega^{a}=0$,

$$
\left.X\right|_{\Omega=0}=0
$$

that it is invariant under background-gauge transformations and satisfies the equation

$$
\Omega^{a} \frac{\delta X}{\delta \phi^{a}}=0
$$

Then there exists a local functional $Y$, invariant under background-gauge transformations, such that

$$
X=\Omega^{a} \frac{\delta Y}{\delta \phi^{a}} .
$$

Proof. The functional $Y$ is constructed explicitly as,

$$
Y=\left(\phi^{a}-\varphi^{a}\right) \frac{\delta}{\delta \Omega^{a}} \int_{0}^{1} \frac{\mathrm{d} z}{z} X[\varphi, \varphi+z(\phi-\varphi), z \Omega, \ldots],
$$

where the arguments of $X$ represented by dots are left untouched. According to the assumption (B.1), this expression indeed provides a regular functional. Notice that if $X$ is local, so is (B.4). Moreover, $Y$ inherits background gauge invariance from $X$ due to the 
linearity of background-gauge transformations. It remains to demonstrate (B.3). Using the anticommutator

$$
\Omega^{a} \frac{\delta}{\delta \phi^{a}}\left(\phi^{b}-\varphi^{b}\right) \frac{\delta}{\delta \Omega^{b}}+\left(\phi^{b}-\varphi^{b}\right) \frac{\delta}{\delta \Omega^{b}} \Omega^{a} \frac{\delta}{\delta \phi^{a}}=\Omega^{a} \frac{\delta}{\delta \Omega^{a}}+\left(\phi^{a}-\varphi^{a}\right) \frac{\delta}{\delta \phi^{a}}
$$

we find

$$
\Omega^{a} \frac{\delta}{\delta \phi^{a}} Y=\int_{0}^{1} \frac{\mathrm{d} z}{z}\left(z \frac{\mathrm{d}}{\mathrm{d} z} X[\varphi, \varphi+z(\phi-\varphi), z \Omega, \ldots]\right)=X[\varphi, \phi, \Omega, \ldots],
$$

where we again used (B.1).

\section{Quadratic form for perturbations in the $O(N)$ model}

Here we summarize the expressions for the coefficients of the operator (6.16) appearing in the quadratic action for the perturbations $\delta \Phi^{I}=\left(\delta \varphi^{i}, \delta \omega, \delta \bar{\omega}\right)$ of the $O(N)$ model. We write these coefficients as matrices with $3 \times 3$ block structure. The coefficient of the d'Alembertian is the (super)symmetric matrix

$$
D_{I J}=\left(\begin{array}{ccc}
A_{i j} & 0 & B_{i} \\
0 & 0 & C \\
-B_{j} & -C & 0
\end{array}\right),
$$

with the following boson-boson $A_{i j}$, boson-fermion $B_{i}$ and fermion-fermion $C$ entries

$$
\begin{aligned}
A_{i j} & =-G_{i j}-\frac{\phi_{i} \phi_{j}}{\left(\phi^{2}\right)^{2}}=-\frac{1}{\varphi^{2}}\left[\delta_{i j}-\frac{\varphi_{i} \varphi_{j}}{\varphi^{2}}+\frac{\varphi^{2}}{\phi^{2}} \frac{\phi_{i} \phi_{j}}{\phi^{2}}\right], \\
B_{i} & =\frac{\phi_{i}}{\phi^{2}} \omega+\frac{\Omega_{i}}{\phi^{2}}-2 \frac{(\Omega \cdot \phi)}{\left(\phi^{2}\right)^{2}} \phi_{i}, \quad C=\frac{\varphi \cdot \phi}{\phi^{2}} .
\end{aligned}
$$

The other two matrix coefficients have the form,

$$
\begin{aligned}
\Gamma_{I J}^{\mu} & =\left(\begin{array}{ccc}
\Gamma_{i j}^{\mu} & 0 & 0 \\
0 & 0 & 0 \\
-\partial^{\mu} B_{j}-\partial^{\mu} C & 0
\end{array}\right), \quad \Pi_{I J}=\left(\begin{array}{ccc}
\Pi_{i j} & \hat{\gamma}_{i} & 0 \\
-\hat{\gamma}_{j} & 0 & 0 \\
-\square B_{j}-\square C & 0
\end{array}\right), \\
\Gamma_{i j}^{\mu} & =-\frac{1}{2}\left(G_{j i, k}+G_{k i, j}-G_{j k, i}\right) \partial^{\mu} \varphi^{k}-\frac{\phi_{i}}{\phi^{2}} \partial^{\mu}\left(\frac{\phi_{j}}{\phi^{2}}\right), \\
\Pi_{i j} & =-\left(G_{i k, j l}-\frac{1}{2} G_{k l, i j}\right) \partial_{\mu} \varphi^{k} \partial^{\mu} \varphi^{l}-G_{k i, j} \square \varphi^{k}-\frac{\phi_{i}}{\phi^{2}} \square\left(\frac{\phi_{j}}{\phi^{2}}\right),
\end{aligned}
$$

where

$$
\begin{aligned}
G_{i j, k} \equiv & \frac{\partial G_{i j}}{\partial \varphi^{k}}=-\frac{2 \delta_{i j} \varphi_{k}+\delta_{i k} \varphi_{j}+\delta_{j k} \varphi_{i}}{\left(\varphi^{2}\right)^{2}}+\frac{4 \varphi_{i} \varphi_{j} \varphi_{k}}{\left(\varphi^{2}\right)^{3}} \\
G_{i j, k l} \equiv & \frac{\partial^{2} G_{i j}}{\partial \varphi^{k} \partial \varphi^{l}}=-\frac{2 \delta_{i j} \delta_{k l}+\delta_{i k} \delta_{j l}+\delta_{j k} \delta_{i l}}{\left(\varphi^{2}\right)^{2}}-\frac{24 \varphi_{i} \varphi_{j} \varphi_{k} \varphi_{l}}{\left(\varphi^{2}\right)^{4}} \\
& +\frac{4}{\left(\varphi^{2}\right)^{3}}\left(2 \delta_{i j} \varphi_{k} \varphi_{l}+\delta_{i k} \varphi_{j} \varphi_{l}+\delta_{i l} \varphi_{j} \varphi_{k}+\delta_{j k} \varphi_{i} \varphi_{l}+\delta_{j l} \varphi_{i} \varphi_{k}+\delta_{k l} \varphi_{i} \varphi_{j}\right) .
\end{aligned}
$$


For the computation of the one-loop divergence of the effective action, we need the matrices $\boldsymbol{\Pi}$ and $\boldsymbol{\Gamma}^{\mu}$ defined in (6.21). This, in turn, requires the inverse $D^{I J}$ of the matrix (C.1), which reads

$$
D^{I J}=\left(\begin{array}{ccc}
A^{i j} & -A^{i k} B_{k} / C & 0 \\
-B_{k} A^{k j} / C & B_{k} A^{k l} B_{l} / C^{2} & -1 / C \\
0 & 1 / C & 0
\end{array}\right),
$$

where $B_{j}$ and $C$ are given by (C.3) and

$$
A^{i j}=-\varphi^{2}\left[\delta^{i j}-\frac{\left(\varphi^{i} \phi^{j}+\phi^{i} \varphi^{j}\right)}{\varphi \cdot \phi}+\frac{\phi^{2}}{\varphi^{2}} \frac{\left(\varphi^{2}+\phi^{2}\right)}{(\varphi \cdot \phi)^{2}} \varphi^{i} \varphi^{j}\right]
$$

is the inverse of the matrix $A_{i j}$ defined by (C.2), $A_{i l} A^{l j}=\delta_{i}^{j}$. Using these expressions we obtain,

$$
\Gamma^{\mu}=\left(\begin{array}{ccc}
A^{i l} \Gamma_{l k}^{\mu} & 0 & 0 \\
\left(\partial^{\mu} B_{k}-B_{l} A^{l m} \Gamma_{m k}^{\mu}\right) / C & \partial^{\mu} C / C & 0 \\
0 & 0 & 0
\end{array}\right)
$$

The diagonal blocks of $\Gamma_{\mu}^{2}$ equal

$$
\left(\boldsymbol{\Gamma}_{\mu}^{2}\right)_{j}^{i}=A^{i l} \Gamma_{l k}^{\mu} A^{k m} \Gamma_{\mu m j}, \quad\left(\boldsymbol{\Gamma}_{\mu}^{2}\right)_{\omega}^{\omega}=\frac{1}{C^{2}}\left(\partial_{\mu} C\right)^{2}, \quad\left(\boldsymbol{\Gamma}_{\mu}^{2}\right)_{\bar{\omega}}^{\bar{\omega}}=0 .
$$

For the matrix of the potential term $\boldsymbol{\Pi}$ we need only its diagonal block elements which read

$$
\boldsymbol{\Pi}_{j}^{i}=A^{i l} \Pi_{l j}+\frac{1}{C} A^{i l} B_{l} \hat{\gamma}_{j}, \quad \boldsymbol{\Pi}_{\omega}^{\omega}=-\frac{1}{C}\left(B_{k} A^{k l} \hat{\gamma}_{l}-\square C\right), \quad \boldsymbol{\Pi}_{\bar{\omega}}^{\bar{\omega}}=0 .
$$

Substituting the above results and expressions for $A^{i j}, B_{i}$ and $C$ into the supertrace of eq. (6.22), str $\boldsymbol{\Pi}=\boldsymbol{\Pi}_{i}^{i}-\boldsymbol{\Pi}_{\omega}^{\omega}-\boldsymbol{\Pi}_{\bar{\omega}}^{\bar{\omega}}$ and similarly for $\operatorname{tr} \boldsymbol{\Gamma}_{\mu}^{2}$, we arrive at eq. (6.23).

Open Access. This article is distributed under the terms of the Creative Commons Attribution License (CC-BY 4.0), which permits any use, distribution and reproduction in any medium, provided the original author(s) and source are credited.

\section{References}

[1] C. Becchi, A. Rouet and R. Stora, Renormalization of the Abelian Higgs-Kibble Model, Commun. Math. Phys. 42 (1975) 127 [InSPIRE].

[2] C. Becchi, A. Rouet and R. Stora, Renormalization of Gauge Theories, Annals Phys. 98 (1976) 287 [InSPIRE].

[3] I.V. Tyutin, Gauge Invariance in Field Theory and Statistical Physics in Operator Formalism (in Russian), Lebedev Institute preprint N39 (1975).

[4] S. Weinberg, The Quantum Theory of Fields. Vol. 2: Modern Applications, Cambridge University Press, Cambridge U.K. (1996). 
[5] K.S. Stelle, Renormalization of Higher Derivative Quantum Gravity, Phys. Rev. D 16 (1977) 953 [InSPIRE].

[6] J. Zinn-Justin, Renormalization of gauge theories, in: Lecture Notes in Physics. Vol. 37: Trends in Elementary Particle Theory, H. Rollnik and K. Dietz eds., Springer, Heidelberg Germany (1975).

[7] B.L. Voronov and I.V. Tyutin, Formulation of gauge theories of general form. I, Theor. Math. Phys. 50 (1982) 218 [Teor. Mat. Fiz. 50 (1982) 333] [InSPIRE].

[8] B.l. Voronov and I.v. Tyutin, Formulation of gauge theories of general form. II. Gauge invariant renormalizability and renormalization structure, Theor. Math. Phys. 52 (1982) 628 [Teor. Mat. Fiz. 52 (1982) 14] [inSPIRE].

[9] D. Anselmi, Removal of divergences with the Batalin-Vilkovisky formalism, Class. Quant. Grav. 11 (1994) 2181 [inSPIRE].

[10] J. Gomis and S. Weinberg, Are nonrenormalizable gauge theories renormalizable?, Nucl. Phys. B 469 (1996) 473 [hep-th/9510087] [INSPIRE].

[11] G. Barnich and M. Henneaux, Renormalization of gauge invariant operators and anomalies in Yang-Mills theory, Phys. Rev. Lett. 72 (1994) 1588 [hep-th/9312206] [INSPIRE].

[12] G. Barnich, F. Brandt and M. Henneaux, Local BRST cohomology in the antifield formalism. II. Application to Yang-Mills theory, Commun. Math. Phys. 174 (1995) 93 [hep-th/9405194] [INSPIRE].

[13] G. Barnich, F. Brandt and M. Henneaux, Local BRST cohomology in Einstein Yang-Mills theory, Nucl. Phys. B 455 (1995) 357 [hep-th/9505173] [INSPIRE].

[14] O. Vafek, Z. Tesanovic and M. Franz, Relativity restored: Dirac anisotropy in QED(3), Phys. Rev. Lett. 89 (2002) 157003 [cond-mat/0203047] [inSPIRE].

[15] M. Franz, Z. Tesanovic and O. Vafek, QED(3) theory of pairing pseudogap in cuprates. 1. From $D$ wave superconductor to antiferromagnet via 'algebraic' Fermi liquid, Phys. Rev. B 66 (2002) 054535 [cond-mat/0203333] [INSPIRE].

[16] E. Ardonne, P. Fendley and E. Fradkin, Topological order and conformal quantum critical points, Annals Phys. 310 (2004) 493 [cond-mat/0311466] [INSPIRE].

[17] B. Roy, V. Juricic and I.F. Herbut, Emergent Lorentz symmetry near fermionic quantum critical points in two and three dimensions, JHEP 04 (2016) 018 [arXiv:1510.07650] [INSPIRE].

[18] S. Kachru, X. Liu and M. Mulligan, Gravity duals of Lifshitz-like fixed points, Phys. Rev. D 78 (2008) 106005 [arXiv:0808.1725] [InSPIRE].

[19] T. Griffin, P. Hořava and C.M. Melby-Thompson, Conformal Lifshitz Gravity from Holography, JHEP 05 (2012) 010 [arXiv:1112.5660] [INSPIRE].

[20] D. Anselmi, Weighted power counting, neutrino masses and Lorentz violating extensions of the Standard Model, Phys. Rev. D 79 (2009) 025017 [arXiv:0808.3475] [INSPIRE].

[21] D. Anselmi, Standard Model Without Elementary Scalars And High Energy Lorentz Violation, Eur. Phys. J. C 65 (2010) 523 [arXiv:0904.1849] [INSPIRE].

[22] R. Iengo and M. Serone, A Simple UV-Completion of QED in 5D, Phys. Rev. D 81 (2010) 125005 [arXiv: 1003.4430] [InSPIRE].

[23] A.O. Barvinsky et al., Heat kernel methods for Lifshitz theories, JHEP 06 (2017) 063 [arXiv: 1703.04747] [INSPIRE]. 
[24] P. Hořava, Membranes at Quantum Criticality, JHEP 03 (2009) 020 [arXiv:0812.4287] [INSPIRE].

[25] P. Hořava, Quantum Gravity at a Lifshitz Point, Phys. Rev. D 79 (2009) 084008 [arXiv:0901.3775] [INSPIRE].

[26] A.O. Barvinsky, D. Blas, M. Herrero-Valea, S.M. Sibiryakov and C.F. Steinwachs, Renormalization of Hořava gravity, Phys. Rev. D 93 (2016) 064022 [arXiv:1512.02250] [INSPIRE].

[27] B.S. DeWitt, Quantum Theory of Gravity. 2. The Manifestly Covariant Theory, Phys. Rev. 162 (1967) 1195 [INSPIRE].

[28] B.S. DeWitt, Quantum Theory of Gravity. 3. Applications of the Covariant Theory, Phys. Rev. 162 (1967) 1239 [inSPIRE].

[29] L.F. Abbott, Introduction to the Background Field Method, Acta Phys. Polon. B 13 (1982) 33 [INSPIRE].

[30] B.S. DeWitt, Dynamical Theory of Groups and Fields, Gordon and Breach, Philadelphia U.S.A. (1965).

[31] M.J.G. Veltman, Quantum Theory of Gravitation, Conf. Proc. C 7507281 (1975) 265 [INSPIRE].

[32] J. Honerkamp, The Question of invariant renormalizability of the massless Yang-Mills theory in a manifest covariant approach, Nucl. Phys. B 48 (1972) 269 [INSPIRE].

[33] G. 't Hooft, An algorithm for the poles at dimension four in the dimensional regularization procedure, Nucl. Phys. B 62 (1973) 444 [INSPIRE].

[34] G. 't Hooft and M.J.G. Veltman, One loop divergencies in the theory of gravitation, Ann. Inst. H. Poincare Phys. Theor. A 20 (1974) 69.

[35] H. Kluberg-Stern and J.B. Zuber, Renormalization of Nonabelian Gauge Theories in a Background Field Gauge. 1. Green Functions, Phys. Rev. D 12 (1975) 482 [InSPIRE].

[36] I.V. Tyutin, Renormalization of the Background Functional in Nonabelian Gauge Theories, Teor. Mat. Fiz. 35 (1978) 29 [inSPIRE].

[37] P.A. Grassi, Stability and renormalization of Yang-Mills theory with background field method: A Regularization independent proof, Nucl. Phys. B 462 (1996) 524 [hep-th/9505101] [INSPIRE].

[38] R. Ferrari, M. Picariello and A. Quadri, Algebraic aspects of the background field method, Annals Phys. 294 (2001) 165 [hep-th/0012090] [INSPIRE].

[39] D. Binosi and A. Quadri, Slavnov-Taylor constraints for non-trivial backgrounds, Phys. Rev. D 84 (2011) 065017 [arXiv:1106.3240] [INSPIRE].

[40] R.E. Kallosh, The Renormalization in Nonabelian Gauge Theories, Nucl. Phys. B 78 (1974) 293 [inSPIRE].

[41] I. Ya. Arefeva, L.D. Faddeev and A.A. Slavnov, Generating Functional for the s Matrix in Gauge Theories, Theor. Math. Phys. 21 (1975) 1165 [Teor. Mat. Fiz. 21 (1974) 311] [INSPIRE].

[42] L.F. Abbott, The Background Field Method Beyond One Loop, Nucl. Phys. B 185 (1981) 189 [inSPIRE]. 
[43] S. Ichinose and M. Omote, Renormalization Using the Background Field Method, Nucl. Phys. B 203 (1982) 221 [inSPIRE].

[44] A.O. Barvinsky and G.A. Vilkovisky, The effective action in quantum field theory: two-loop approximation, in Quantum Field Theory and Quantum Statistics. Vol. 1, I. Batalin, C.J. Isham and G.A. Vilkovisky eds., Hilger, Bristol U.K. (1987), pg. 245.

[45] D. Anselmi, Background field method, Batalin-Vilkovisky formalism and parametric completeness of renormalization, Phys. Rev. D 89 (2014) 045004 [arXiv:1311.2704] [INSPIRE].

[46] D. Binosi and A. Quadri, Canonical Transformations and Renormalization Group Invariance in the presence of Non-trivial Backgrounds, Phys. Rev. D 85 (2012) 085020 [arXiv: 1201.1807] [INSPIRE].

[47] D. Binosi and A. Quadri, The Background Field Method as a Canonical Transformation, Phys. Rev. D 85 (2012) 121702 [arXiv:1203.6637] [InSPIRE].

[48] I.A. Batalin and G.A. Vilkovisky, Gauge Algebra and Quantization, Phys. Lett. B 102 (1981) 27.

[49] I.A. Batalin and G.A. Vilkovisky, Feynman Rules For Reducible Gauge Theories, Phys. Lett. B 120 (1983) 166.

[50] I.A. Batalin and G.A. Vilkovisky, Quantization of Gauge Theories with Linearly Dependent Generators, Phys. Rev. D 28 (1983) 2567 [Erratum ibid. D 30 (1984) 508] [INSPIRE].

[51] I.A. Batalin and G.A. Vilkovisky, Existence Theorem for Gauge Algebra, J. Math. Phys. 26 (1985) 172 [INSPIRE].

[52] D. Anselmi, Weighted power counting and Lorentz violating gauge theories. I. General properties, Annals Phys. 324 (2009) 874 [arXiv:0808.3470] [INSPIRE].

[53] D. Anselmi, Weighted power counting and Lorentz violating gauge theories. II. Classification, Annals Phys. 324 (2009) 1058 [arXiv:0808.3474] [INSPIRE].

[54] P. Hořava, Quantum Criticality and Yang-Mills Gauge Theory, Phys. Lett. B 694 (2011) 172 [arXiv: 0811.2217] [INSPIRE].

[55] J. Wess and J. Bagger, Supersymmetry and Supergravity, Princeton University Press, Princeton U.S.A. (1983).

[56] M. Henneaux, Space-time Locality of the BRST Formalism, Commun. Math. Phys. 140 (1991) 1 [INSPIRE].

[57] J.C. Collins, Renormalization, Cambridge University Press, Cambridge U.K. (1984).

[58] D. Anselmi and M. Halat, Renormalization of Lorentz violating theories, Phys. Rev. D 76 (2007) 125011 [arXiv:0707.2480] [INSPIRE].

[59] K.S. Stelle, Classical Gravity with Higher Derivatives, Gen. Rel. Grav. 9 (1978) 353 [INSPIRE].

[60] S. Vandoren and A. Van Proeyen, Simplifications in Lagrangian BV quantization exemplified by the anomalies of chiral W(3) gravity, Nucl. Phys. B 411 (1994) 257 [hep-th/9306147] [INSPIRE].

[61] A.M. Polyakov, Gauge Fields and Strings, Harwood Academic Publishers, Reading U.K. (1987). 